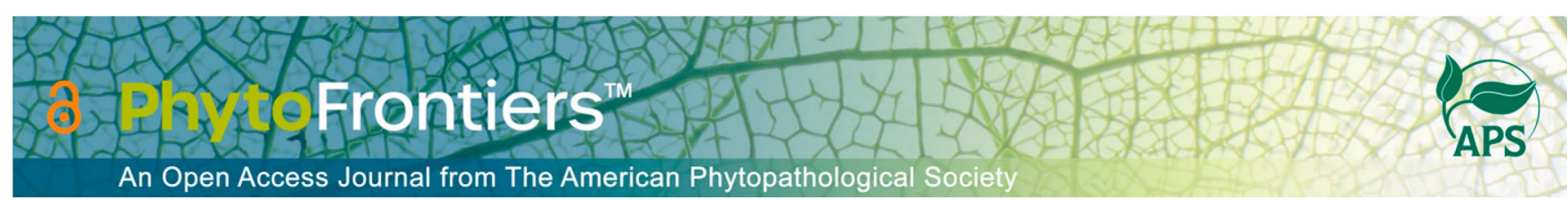

\title{
Research
}

\section{Effects of Wheat Cultivar Mixtures on Population Genetic Structure of Puccinia striiformis f. sp. tritici}

\author{
Bingyao Chu (1) | Kai Yuan | Cuicui Wang (1) | Lujia Yang | Bingbing Jiang | Yilin Gu | Zhanhong $\mathrm{Ma}^{+}$|
}

Department of Plant Pathology, MARA Key Lab of Pest Monitoring and Green Management, College of Plant Protection, China Agricultural University, Beijing 100193, China

† Corresponding author: Z. Ma; mazh@cau.edu.cn Accepted for publication 6 May 2021

\section{Funding}

Support was provided by the National Key R and D Program of China (2017YFD0200400 and 2017YFD0201700), the Key research and development projects of Ningxia Hui autonomous region (East and West Science and Technology Cooperation Project 2017BY080), and National Natural Science Foundation of China (32001841).

$\boldsymbol{e}$-Xtra: Supplementary materials are available online.

The author(s) declare no conflict of interest.

\begin{abstract}
Wheat stripe rust, caused by Puccinia striiformis f. sp. tritici(Pst), is one of the most destructive wheat diseases worldwide. Understanding the effects of cultivar mixture on disease control and population genetic structure of Pst could promote the effective application of cultivar mixtures. This study designed nine cultivar mixtures composed of different component cultivars with field experiments in Zitong County, Sichuan Province in two seasons from 2016 to 2018. Diseased leaves were collected in each plot of treatment in the spring and, in total, 835 and 568 isolates were obtained and genotyped with 10 pairs of simple sequence repeat markers in the 2 years, respectively. The observed and theoretical values of the area under the disease progress curve (AUDPC), wheat yield, Pst genotypic diversity, and the frequency of $P$ st dominant genetic groups were compared among cultivar mixtures. With the 2 years' experiments, there were three and two cultivar mixtures, respectively, showing significant effect on the relative reduction of AUDPC; there were no significant effects on the relative yield increase in all mixtures in the 2 years. There were four and five cultivar mixtures showing positive effects on the relative increase of Pst genotypic diversity in the 2 years, respectively. Moreover, all cultivar mixtures could decrease the frequencies of Pst dominant genetic groups on highly susceptible component cultivar compared with their theoretical frequencies.
\end{abstract}

Keywords: cultivar mixture, genetic structure, population diversity, Puccinia striiformis f. sp. tritici, wheat

Stripe rust, caused by Puccinia striiformis f. sp. tritici (Pst) is one of the most important diseases of wheat in the world (Chen 2005; Savary et al. 2019; Wan et al. 2007). The major strategies for the management of wheat stripe rust still relay on the applications of resistant varieties and fungicides in China (Wan et al. 2007). However, using a single, highly resistant variety on a large scale imposes high directional selection pressure on Pst populations (Bahri et al. 2011; McDonald and Linde 2002; Zhan et al. 2013; J. Zhan et al. 2015). The rapid evolution of $P s t$ populations threatens currently planted wheat cultivars at the risk of resistance losses and severe disease epidemics 
(Li and Zeng 2002; Luo et al. 2007). Therefore, cultivar mixtures, as a new cropping strategy to imitate the natural ecological system and to reduce the directional selection on pathogen populations, have been studied over 60 years and are increasingly used for disease control (Finckh and Wolfe 2006; Finckh et al. 2000; Mundt 2002; Zhu et al. 2000).

Pst is an obligate biotrophic pathogen with a feature of parasitic specialization (Wan 2003). The five severe national pandemics of wheat stripe rust and seven periods of significant resistance losses in China were all closely related to the change in population structure of Pst (Kang et al. 2015; Li and Zeng 2002; Wan et al. 2007). Recently, the physiological race CYR34 and pathogenic group Guinong 22 of Pst have risen to be dominant. They can infect wheat cultivars with $Y r 26$ and $\mathrm{YrCH} 42$ genes and seriously threaten wheat production in China (Liu et al. 2010; Liu et al. 2017). Therefore, it is important for disease management to study the effect of cultivar mixtures on population genetic structure of $P$ st.

The effects of cultivar mixtures on the diversity of pathogen populations have been studied extensively (Zhu et al. 2004a, 2000). Müller et al. (1996) found that the diversity of Erysiphe graminis f. sp. hordei pathotypes in barley was generally related to the degree of diversification of host resistance in time and space. Yang et al. (2004) revealed that the constitution of the physiological race of Magnaporthe grisea in a field of rice cultivar mixture tended to be more diverse compared with monoculture. He et al. (2003) and Zhang et al. (2004) found an increase in the number of genetic groups and a decrease in the frequency of the dominant genetic group of $M$. oryzae in fields of rice cultivar mixtures, determined by repetitive element sequence-based PCR molecular fingerprint technology; Jia (2007) obtained a similar result in the wheat-Blumeria graminis f. sp. tritici pathosystem using one intersimple sequence repeat primer. Guo (2012) found that the percentage of polymorphic loci and genetic diversity of $P s t$ were higher in wheat cultivar mixtures than in monocultures using five pairs of simple sequence repeat (SSR) primers. These studies concluded that pathogen populations were more diverse in cultivar mixtures than in monocultures. However, because the sample sizes or primer numbers in these studies were insufficient, the results might not fully reflect the actual situations in the fields. Moreover, the relative genotypic diversities of Pst and frequencies of $P s t$ dominant genetic groups in cultivar mixtures are unclear. We might expect greater pathogen diversity in mixtures of multiple hosts, even if the host diversity does not influence the pathogen population. The real question is how the diversity and frequency of Pst dominant genetic groups change compared with the theoretical values we expect. Thus, improved experimental techniques and data analysis methods are needed. In this study, we determined the effects of cultivar mixtures on the relative genotypic diversity and frequency of Pst dominant genetic groups in a large sample size.

Although the effect of cultivar mixtures on small grain foliar diseases caused by airborne pathogens has been determined, substantial variations in disease control efficacies exist among cultivar mixtures (Mundt 2002). Disease reduction owing to cultivar mixtures relative to monocultures might range from 4 to 89\% (Smithson and Lenne 1996). In 11 studies of wheat stripe rust involving 161 cultivar mixtures, approximately $83 \%$ of cultivar mixtures had positive effects on disease reduction, with a 30 to $50 \%$ reduction in control efficacies in most cases (Huang et al. 2012). Factors affecting the cultivar mixture effects on disease control include mixture composition, epidemic intensity, sowing density, and spatial scale of field (Garrett and Mundt
1999; Mundt 2002; Mundt et al. 1995a). Specifically, mixture composition refers to the number of components (Q. Chen et al. 2009; Guo 2012; Mundt 1994; Newton et al. 1997), the resistance level of the components (Guo 2012), the genetic background of the components (Zhu et al. 2004b), and plant interactions among component cultivars in the mixtures (Finckh and Mundt 1992a, b). Comparing mixture effects with various cultivar mixtures under these different factors could help us understand the effects of cultivar mixtures on disease control and $P$ st population genetic structure.

The objectives of this study were to (i) determine the relative efficacies of cultivar mixtures on disease control of wheat stripe rust and yield increase of seed with two-year experiments, (ii) demonstrate the effects of cultivar mixtures on the diversity and genetic structure of Pst populations, and (iii) compare the differences in cultivar mixture effects and Pst populations with various cultivar mixtures.

\section{MATERIALS AND METHODS}

\section{Experimental design}

A cultivar mixture experimental field was selected at Dongshi Village, Zitong County, located in the Sichuan Basin of Sichuan Province $\left(\mathrm{N}: 31^{\circ} 34^{\prime}, \mathrm{E}: 105^{\circ} 10^{\prime}\right)$. This area is the winter-wheatgrowing area in China, where the wheat is planted at the end of October and harvested in the middle of May. Due to the yearround high humidity and warm temperature, $P$ st can overwinter there and the disease can frequently occur by natural infections. Five wheat cultivars (A: Miannong 4 hao, B: Neimai 9 hao, C: Chuanmai 51, D: Chuanyu 21 and E: Chuanmai 55) were selected among 100 cultivars from Sichuan Province based on features including growth period and different infection types (Chu et al. 2019; Stubbs 1985) to Pst (Table 1). Nine cultivar mixture treatments assigned as M1 to M9 were generated by consideration of combinations of various cultivars with different resistance levels (Table 1). The seed of all component cultivars in mixtures were mixed in equal proportions.

In the field, each plot was $4 \mathrm{~m}$ long and $4 \mathrm{~m}$ wide with 16 rows and $0.25 \mathrm{~m}$ of space between rows. The planting density was $16 \mathrm{~g} / \mathrm{m}^{2}$ (approximately 300 seeds $/ \mathrm{m}^{2}$ ). There were 42 plots which were separated with resistant cultivars (Nongda 211) at $2 \mathrm{~m}$ in width to prevent contaminations. The field experiment was arranged as a complete block design with three replications. Experiments with the same design were conducted in 2 years from 2016 to 2018. During the whole growing period of wheat, standard water and fertilizer management was carried out, and no growth regulators or pesticides were applied.

\section{Pst sampling and isolation}

Pst isolates were collected from all plots including pure stands and cultivar mixtures on 28 March 2017 and 30 March 2018. In each plot, 100 to 150 leaves were randomly collected from diseased leaves. Samples from three replications of a treatment in a year were combined and considered as one population; thus, 14 populations were obtained each year. Leaves showing sporulation were separately stored in paper bags, and dried in desiccators at $4^{\circ} \mathrm{C}$ for later isolation.

A single pustule was used to obtain one isolate based on the assumption that each pustule was caused by a single urediniospore. Sampled leaves were placed on the moist filter papers 
in petri dishes at $9^{\circ} \mathrm{C}$ for 10 to $15 \mathrm{~h}$ in darkness. The spores from the single pustule on a sampled leaf were picked and rubbed in a drop of water on the leaf surface of universally susceptible cultivar Mingxian 169 as inoculation. The inoculated plants were incubated in dew chamber at $10^{\circ} \mathrm{C}$ for $24 \mathrm{~h}$ in darkness, then transferred to the growth chamber at 10 to $15^{\circ} \mathrm{C}$ with 60 to $70 \%$ relative humidity $(\mathrm{RH})$ and a photoperiod of 14 of light and $10 \mathrm{~h}$ of darkness (10,000 lux). Approximately 13 to 15 days after inoculation, urediniospores were harvested from the sporulating lesions to a tube, and dried in desiccators at $4{ }^{\circ} \mathrm{C}$ for 3 days. In cultivar mixtures, it was difficult to identify the particular mixture component cultivar from which the isolations were made; therefore, only one single pustule was isolated from each leaf to ensure that the amount of Pst isolates sampled from different components was consistent with their disease incidences. Each isolate was propagated for one to two generations as described above to obtain an adequate amount of urediniospores. Urediniospores were transferred into a 0.5-ml Eppendorf tube and stored at $-80^{\circ} \mathrm{C}$ for later DNA extraction. The information of obtained isolates is shown in Table 1.

\section{DNA extraction and SSR amplification}

Genomic DNA of each isolate was extracted by following Wan et al. (2015). The concentration of each DNA sample was determined via UV absorption at wavelengths of 260 and $280 \mathrm{~nm}$ using a Nanodrop 2000 Spectrophotometer (Gene Company Limited, China). The DNA samples were diluted to the concentration of $100 \mathrm{ng} / \mu \mathrm{l}$ and stored at $-20^{\circ} \mathrm{C}$ for $\mathrm{SSR}$ amplification.

Ten pairs of published SSR primers were used to genotype each isolate (Table 2), with three corresponding PCR systems (Supplementary Table S1). All SSR primers were modified by FAM, HEX, ROX, or TAMRA fluorescence at the $5^{\prime}$ end. All of the SSR amplifications were performed in an Authorized Thermal Cycler (Eppendorf AG). Subsequent separation of the PCR products was conducted using the ABI $3730 \mathrm{xl}$ (Applied Biosystems) DNA Analyzer, and DNA Marker GS500 (35 to $500 \mathrm{bp}$ ) was used as an internal standard. GeneMarker software (version 2.4.0; SoftGenetics, LLC, State College, PA, U.S.A.) was used to read the band results.

\section{Disease assessment and yield recording}

Disease assessment was conducted with a five-site sampling method (one site was in the center and the other four sites were $2 \mathrm{~m}$ away from center in the direction of four corners; a sign was stuck in the ground of each site and the disease assessment range of each site covered two rows of wheat, each $30 \mathrm{~cm}$ in length) for each plot in the field. In each sampling site, the

\section{TABLE 1}

Information about cultivars used in this study and the number of Puccinia striiformis $\mathrm{f}$. sp. tritici isolates and genotypes sampled in each monoculture and cultivar mixture ${ }^{\mathrm{a}}$

\begin{tabular}{|c|c|c|c|c|c|c|c|c|}
\hline \multirow[b]{2}{*}{ Treatment } & \multirow[b]{2}{*}{ Sources } & \multicolumn{2}{|c|}{$2016-17$} & \multicolumn{2}{|c|}{$2017-18$} & \multirow{2}{*}{$\begin{array}{l}\text { Height } \\
(\mathrm{cm})\end{array}$} & \multirow[b]{2}{*}{ Growing period (day) } & \multirow[b]{2}{*}{ IT } \\
\hline & & $N$ & $N G$ & $N$ & $N G$ & & & \\
\hline Miannong 4 hao (A) & 75-21-4/76-19//Miannong 1 hao & 53 & 17 & 35 & 7 & 85 & $185-188$ & 4 \\
\hline Neimai 9 hao (B) & Mianyang 26/92R178 & 67 & 21 & 39 & 19 & 84 & 190 & 3 \\
\hline Chuanmai 51 (C) & 174/183//99-1572 & 67 & 26 & 42 & 18 & 100 & 183 & 2 \\
\hline Chuanyu 21 (D) & Zhou 88114/G159 & 60 & 41 & 33 & 11 & 90 & 186 & 1 \\
\hline Chuanmai 55 (E) & SW3243/SW8688 & 55 & 17 & 24 & 6 & 83 & 185 & 1 \\
\hline M1 & $\mathrm{A}+\mathrm{D}+\mathrm{E}$ & 48 & 25 & 44 & 8 & - & - & - \\
\hline M2 & $\mathrm{A}+\mathrm{C}+\mathrm{D}$ & 73 & 32 & 40 & 15 & - & - & - \\
\hline M3 & $\mathrm{A}+\mathrm{B}+\mathrm{C}$ & 86 & 43 & 47 & 21 & - & - & - \\
\hline M4 & $\mathrm{B}+\mathrm{D}+\mathrm{E}$ & 43 & 26 & 40 & 17 & - & - & - \\
\hline M5 & $\mathrm{C}+\mathrm{D}+\mathrm{E}$ & 47 & 27 & 42 & 21 & - & - & - \\
\hline M6 & $\mathrm{B}+\mathrm{C}+\mathrm{D}$ & 57 & 26 & 48 & 30 & - & - & - \\
\hline M7 & $\mathrm{B}+\mathrm{C}+\mathrm{E}$ & 59 & 20 & 39 & 22 & - & - & - \\
\hline M8 & $\mathrm{B}+\mathrm{C}+\mathrm{D}+\mathrm{E}$ & 59 & 31 & 48 & 28 & - & - & - \\
\hline M9 & $\mathrm{A}+\mathrm{B}+\mathrm{C}+\mathrm{D}+\mathrm{E}$ & 61 & 27 & 47 & 21 & - & - & - \\
\hline
\end{tabular}

${ }^{\mathrm{a}} N=$ the number of isolates and $N G=$ the number of genotypes. Infection types (ITs) 3 and 4 represent susceptible and 1 and 2 represent resistant to stripe rust (Chu et al. 2019; Stubbs 1985).

TABLE 2

Information on the simple sequence repeat primers for Puccinia striiformis $\mathrm{f}$. sp. tritici used in this study

\begin{tabular}{|c|c|c|c|c|}
\hline \multirow[b]{2}{*}{ Locus } & \multicolumn{2}{|c|}{ Sequence $\left(5^{\prime}-3^{\prime}\right)$} & \multirow[b]{2}{*}{ Size $^{\mathrm{a}}$} & \multirow[b]{2}{*}{ Reference } \\
\hline & Forward primer & Reverse primer & & \\
\hline CPS8 & GATAAGAAACAAGGGACAGC & CAGTGAACCCAATTACTCAG & $200-209$ & C. Chen et al. 2009 \\
\hline CPS13 & TCCAGGCAGTAAATCAGACGC & ATCAGCAGGTGTAGCCCCATC & $125-128$ & C. Chen et al. 2009 \\
\hline CPS34 & GTTGGCTACGAGTGGTCATC & TAACACTACACAAAAGGGGTC & $107-113$ & C. Chen et al. 2009 \\
\hline RJ3N & TGGTGGTGCTCCTCTAGTC & AGGGGTCTTGTAAGATGCTC & $338-346$ & Bahri et al. 2009 \\
\hline RJ5N & AACGGTCAACAGCACTCAC & AGTTGGTCGCGTTTTGCTC & $222-227$ & Bahri et al. 2009 \\
\hline RJ6N & CAATCTGGCGGACAGCAAC & CACCTAGGATACCACCGCC & $309-342$ & Bahri et al. 2009 \\
\hline RJ13N & TTAGCTCAGCCGGTTCCTC & CAGGTGTAGCCCCATCTCC & $143-152$ & Bahri et al. 2009 \\
\hline RJO3 & GCAGCACTGGCAGGTGG & GATGAATCAGGATGGCTCC & 195-205 & Enjalbert et al. 2002 \\
\hline RJO20 & AGAAGATCGACGCACCCG & CCTCCGATTGGCTTAGGC & $283-289$ & Enjalbert et al. 2002 \\
\hline WSR44 & AGGCCCCAGGAACACAAAAA & TCACACACGCTCCACAGTAC & $187-189$ & G. Zhan et al. 2015 \\
\hline
\end{tabular}

${ }^{\mathrm{a}}$ Allele size range. 
assessors entered the plots and stood between the rows, and 25 to 30 plants (approximately 100 leaves) were randomly selected to record the number of diseased leaves and disease severities by visual inspection. The disease severity was assessed as the percentage of lesion area over the whole leaf area, which was classified into eight levels of $1,5,10,20,40,60,80$, and $100 \%$ (Shang et al. 1990). The disease index (DI) was calculated as the average disease severity $\times$ the disease incidence (the number of diseased leaves/total number of leaves assessed) $\times 100$. Wheat growth stages were recorded according to Zadoks et al. (1974). Disease assessments were conducted four times in 2017, on 17 January (GS21), 17 February (GS28), 1 March (GS39), and 21 March (GS49); and three times in 2018, on 19 March (GS45), 29 March (GS60), and 12 April (GS71). At harvest, yield of every plot (in kilograms) was obtained. The wheat moisture content was measured by a grain moisture analyzer and a wheat weight with $13 \%$ water content was obtained (Xing and Tian 2011). The average wheat yield for each treatment was obtained from three replications for analysis.

\section{Data analysis}

The area under the disease progress curve (AUDPC) of the DI over the disease assessment time points was calculated for each plot as follows:

$$
\mathrm{AUDPC}=\sum_{i}^{n-1}\left[\left(\frac{x_{i}+x_{i+1}}{2}\right)\left(t_{i+1}-t_{i}\right)\right]
$$

where $\mathrm{n}$ is the total number of disease assessment time points; $x_{i}$ and $x_{i+1}$ are the DI at $i$ th and $(i+1)$ th disease assessments, respectively; and $t_{i}$ and $t_{i+1}$ are days of the disease progress curve at $i$ th and $(i+1)$ th disease assessments, respectively. The theoretical AUDPC and yield for each cultivar mixture were calculated by averaging the observed AUDPC and yield of corresponding components in monocultures to determine the effect of cultivar mixtures on disease control and yield increase. The one-sample $t$ test (McDonald 2014a) was used to determine whether the observed AUDPC and wheat yield in each mixture differed significantly from its corresponding theoretical AUDPC and yield using SPSS version 20.0 (IBM). A test was considered significant at $P<0.0056$ based on the Bonferroni correction by dividing $P=0.05$ by the number of tests. The relative efficacies on disease control and yield increase for each plot were estimated as $\left[\left(a_{2}-a_{1}\right) / a_{2}\right] \times 100 \%$, where $a_{1}$ is the observed AUDPC (or yield) of cultivar mixture and $a_{2}$ is the corresponding theoretical value. Welch's $t$ test (McDonald 2014b) was conducted to determine the difference in relative disease control efficacies between the cultivar mixture groups of M1, M2, M3, and M9 that included the highly susceptible cultivar A and M4, M5, M6, M7, and M8 that did not include the highly susceptible cultivar A in the 2-year study period. Due to the heterogeneity of variance, the relative disease control efficacy data were arcsine-square root transformed and used for analysis. Next, analysis of variance (McDonald 2014c) and Tukey's honestly significant difference test (Tukey 1953) were conducted to determine the significance of the difference in relative disease control efficacy and relative yield increase efficacy among the different mixtures using SPSS version 20.0 (IBM). The Pearson correlation analysis (Benesty et al. 2009) was conducted to determine the correlation between relative disease control efficacy and relative yield increase efficacy of the cultivar mixtures using SPSS version 20.0 (IBM).
The genotypic diversity was evaluated by $(i)$ the proportion of multilocus genotypes $(P G)$, also known as genotypes richness, which was calculated by dividing the number of genotypes $(s)$ by the total number of individuals $(n)$; (ii) genotype evenness $(E)$, which was calculated as $-\left(\sum_{r}^{s} P_{r} \ln P_{r}\right) / \ln s$ on the basis of frequencies of genotypes $P_{r}, r=1,2, \ldots, s$ (Sheldon 1969); and (iii) Shannon's diversity index $(S h)$, which was calculated as $-\left(\sum_{r}^{s} P_{r} \ln P_{r}\right) / \ln n$ and satisfied the richness and evenness of genotypic diversity (Andrivon and de Vallavieille-Pope 1995; Goodwin et al. 1992). Distinct genotypes in populations were obtained with GeneClone 2.0 program (Arnaud-Haond and Belkhir 2007). $E$ and $S h$ were obtained by VAT software (Schachtel et al. 2012). To evaluate the effect of different cultivar mixtures on the genotypic diversity of $P s t$, the relative increase in genotypic diversity of $P s t$ in cultivar mixture was estimated as $\left[\left(b_{1}-b_{2}\right) / b_{2}\right] \times 100 \%$, where $b_{1}$ is the observed genotypic diversity $(P G, E$, and $S h)$ in cultivar mixture and $b_{2}$ is the corresponding theoretical genotypic diversity $(P G, E$, and $S h)$. The theoretical genotypic diversity was predicted based on the condition that the cultivar diversity in mixture did not influence the $P s t$ population (i.e., Pst populations increased independently on the different cultivars in mixture). Due to the fact that different monocultures could select for the same or different Pst genotypes, genotypes from different components should be put together for comparison, and the theoretical genotypic diversities of Pst in cultivar mixtures were manually calculated by following the formulas of $P G, E$, and $S h$ based on the prediction of $s$ and $P r$ in mixtures. A reference example is described in Table 3 to show the data processing for the calculation of theoretical genotypic diversity of $P$ st in cultivar mixtures.

To test the effect of different cultivar mixtures on the adjustment of Pst genotypes, the analysis of molecular variance (AMOVA) was conducted by GenALEx version 6.5 (Peakall and Smouse 2006, 2012) to examine the genotypic variation among $P$ st populations from 5 pure stands, 9 cultivar mixtures, and 14 total treatments. PhiPT, an analog of Fst, was obtained based on the codominant genotypic distance matrices with 999 permutations to estimate the pairwise genotypic variation between populations of five pure stands, and populations of cultivar mixtures and their component monocultures. Also, principal coordinate analysis (PCoA) was conducted to show the distribution of genotypes among populations of pure stands, and cultivar mixtures and their component monocultures.

To evaluate the effect of cultivar mixtures on the frequency of Pst dominant genetic groups, the genetic structuring of individuals in each year was determined by two method: the unweighted pair-group method with arithmetic mean (UPGMA) cluster implemented in NTsys version 2.11c (Jensen 1989) and model-based Bayesian cluster implemented in STRUCTURE 2.3.4 (Pritchard et al. 2000). The codominant SSR data were converted to a binary data matrix by treating absence as 0 and presence as 1 of a defined allele. An unbiased estimate of pairwise genetic distances between individuals by simple match coefficient was obtained based on the binary data using NTsys version 2.11c (Jensen 1989), and two phylogenetic trees were constructed with 2 years of isolates using the UPGMA method. The stability of tree branches was tested by cophenetic correlation analysis (1,000 times). Thus, the number of genetic groups and the number of individuals in each genetic group for each population could be determined when a genetic similarity coefficient was determined. The frequency of each genetic group for each population was calculated by dividing the number of the individuals in each genetic group by the total number of individuals. 
For the model-based Bayesian cluster implemented in STRUCTURE 2.3.4 (Pritchard et al. 2000), all of the Pst isolates were assigned into $K$ clusters ranging from 1 to 10 . For each simulated cluster $K, 50$ independent runs were performed with 40,000 iterations in a Monte Carlo Markov Chain scheme after an initial burn-in period of 100,000. The best $K$ value was evaluated in the website of STRUCTURE HARVESTER (http:// taylor0.biology.ucla.edu/structureHarvester) indicated by $\Delta K$ as proposed by Evanno et al. (2005). Indi files and pop files of the best $K$ were processed with CLUMPP 1.1.2 (Jakobsson and Rosenberg 2007); then, Distruct 1.1 (Rosenberg 2004) was used to generate a bar plot using the output of CLUMPP 1.1.2 (Jakobsson and Rosenberg 2007). Individuals were partitioned into multiple groups based on the membership fraction $\mathrm{Q}(\mathrm{Q}>0.8)$ (Pritchard et al. 2000). Thus, the number of individuals assigned to each cluster could be obtained and the percentage of individuals assigned to each cluster for each population was calculated. The relative decrease in frequency of Pst dominant genetic groups of each component monoculture in cultivar mixture was estimated as $\left[\left(c_{2}-c_{1}\right) / c_{2}\right] \times 100 \%$, where $c_{1}$ is the observed frequency of $P s t$ dominant genetic groups in the cultivar mixture obtained by NTsys or STRUCTURE and $c_{2}$ is the corresponding theoretical frequency of Pst dominant genetic groups. The calculation of theoretical frequency of dominant genetic group of Pst was similar to the theoretical frequency of genotype of Pst in the cultivar mixture (Table 3). It was estimated as $\sum_{i}^{n} P_{i} x_{i} / \sum_{i}^{n} x_{i}$, where $n$ is the number of cultivars that included in cultivar mixture, $P_{i}$ is the frequency of $P s t$ dominant genetic groups in $i$ th component monoculture obtained by NTsys or STRUCTURE, and $x_{i}$ is the disease incidence of $i$ th component monoculture on 21 March for the 2016-17 season and 29 March for the 2017-18 season.

\section{RESULTS}

\section{Effects of cultivar mixtures on disease and yield}

Based on the AUDPC, cultivars A, B, C, D, and E were highly susceptible, moderately susceptible, moderately resistant, highly resistant, and highly resistant, respectively, to Pst in the 2016-17 experiment. Cultivar A was highly susceptible and cultivars B, C, D, and E were resistant to Pst in the 2017-18 experiment. The AUDPCs of cultivar mixtures M4, M6, and M7 in 2016-17 and M1 and M3 in 2017-18 were significantly lower than their corresponding theoretical values $(P<0.0056)$

\section{TABLE 3}

Reference example for the calculation method of theoretical genotypic diversity proportion of multilocus genotypes $(P G)$, genotype evenness $(E)$, and Shannon's diversity index (Sh) of Puccinia striiformis f. sp. tritici (Pst) in a cultivar mixture ${ }^{\mathrm{a}}$

\begin{tabular}{|c|c|c|c|c|c|c|c|c|c|c|c|}
\hline \multirow[b]{2}{*}{ Genotype } & \multicolumn{3}{|c|}{ Number of genotypes in monocultures } & \multicolumn{3}{|c|}{$\begin{array}{l}\text { Frequency of genotypes in } \\
\text { monocultures }\end{array}$} & \multirow[b]{2}{*}{$\mathrm{TF}^{\mathrm{c}}$} & \multirow[b]{2}{*}{$\mathrm{TN}$} & \multirow[b]{2}{*}{$\mathrm{TN}^{\prime \mathrm{d}}$} & \multirow[b]{2}{*}{$\mathrm{TN}^{\prime \prime \mathrm{e}}$} & \multirow[b]{2}{*}{$\operatorname{Pr}^{\mathrm{f}}$} \\
\hline & $\mathrm{C} 1$ & $\mathrm{C} 2$ & $\mathrm{C} 3$ & $\mathrm{C} 1$ & $\mathrm{C} 2$ & $\mathrm{C} 3$ & & & & & \\
\hline g1 & 16 & - & 1 & 0.47 & 0.00 & 0.04 & 0.32 & 9.54 & $9(0.54)$ & $9+1$ & 0.33 \\
\hline g2 & 6 & 1 & - & 0.18 & 0.03 & 0.00 & 0.12 & 3.74 & $7(0.48)$ & 4 & 0.14 \\
\hline g3 & 6 & 1 & - & 0.18 & 0.03 & 0.00 & 0.12 & 3.74 & - & 3 & 0.10 \\
\hline $\mathrm{g} 4$ & 3 & 5 & 2 & 0.09 & 0.16 & 0.08 & 0.10 & 3.10 & $3(0.10)$ & 3 & 0.10 \\
\hline g5 & 2 & - & 5 & 0.06 & 0.00 & 0.19 & 0.06 & 1.82 & $1(0.82)$ & $1+1$ & 0.07 \\
\hline g6 & 1 & 12 & - & 0.03 & 0.39 & 0.00 & 0.11 & 3.17 & $3(0.17)$ & 3 & 0.10 \\
\hline g7 & - & 6 & - & 0.00 & 0.19 & 0.00 & 0.04 & 1.29 & $1(0.29)$ & 1 & 0.03 \\
\hline g8 & - & 4 & 2 & 0.00 & 0.13 & 0.08 & 0.04 & 1.12 & $1(0.12)$ & 1 & 0.03 \\
\hline g9 & - & 1 & 5 & 0.00 & 0.03 & 0.19 & 0.03 & 0.86 & $2(0.92)$ & $2+1$ & - \\
\hline $\mathrm{g} 10$ & - & 1 & 1 & 0.00 & 0.03 & 0.04 & 0.01 & 0.34 & - & - & - \\
\hline $\mathrm{g} 11$ & - & 1 & & 0.00 & 0.03 & 0.00 & 0.01 & 0.22 & - & - & 0.03 \\
\hline $\mathrm{g} 12$ & - & 1 & 3 & 0.00 & 0.03 & 0.12 & 0.02 & 0.60 & - & - & 0.03 \\
\hline $\mathrm{g} 13$ & - & - & 5 & 0.00 & 0.00 & 0.19 & 0.02 & 0.64 & - & - & 0.03 \\
\hline $\mathrm{g} 14$ & - & - & 1 & 0.00 & 0.00 & 0.04 & 0.00 & 0.13 & - & - & - \\
\hline g15 & - & - & 1 & 0.00 & 0.00 & 0.04 & 0.00 & 0.13 & - & - & - \\
\hline Sum & 34 & 31 & 26 & - & - & - & - & - & - & 30 & - \\
\hline
\end{tabular}

${ }^{a}$ Hypothesis: this cultivar mixture was composed of three wheat cultivars (C1, C2, and C3) in equal proportions. There were 34, 31, 26, and 30 isolates sampled in monocultures of cultivars $\mathrm{C} 1, \mathrm{C} 2, \mathrm{C} 3$, and this cultivar mixture, respectively. Fifteen genotypes were obtained from monocultures of C1, C2, and $\mathrm{C} 3$, coded as $\mathrm{g} 1$ to $\mathrm{g} 15$. Disease incidences of monocultures of $\mathrm{C} 1, \mathrm{C} 2$, and $\mathrm{C} 3$ were $0.6,0.2$, and 0.1 , respectively. Thus, the frequency of the 15 genotypes in monocultures of $\mathrm{C} 1, \mathrm{C} 2$, and $\mathrm{C} 3$ could be determined. In this study, diseased leaves from different components in cultivar mixtures were collected by random sampling and only one pustule was isolated in each leaf; therefore, the amount of $P$ st stains from different components in mixture were considered to be basically consistent with their disease incidences. The theoretical frequency of each genotype (TF) in cultivar mixture could be calculated as $\sum_{i}^{n} P_{i} x_{i} / \sum_{i}^{n} x_{i}$, where $n$ is the number of cultivars that included in mixture, $P_{i}$ is the frequency of $P s t$ genotype in $i$ th component monoculture, and $x_{i}$ is the disease incidence of $i$ th component monoculture on 21 March for the 2016-17 season and 29 March for the 2017-18 season. The theoretical number of each genotype in a cultivar mixture (TN) was obtained by multiplying TF by the total number of individuals $(n=30)$. The TN was divided into integer and decimal part, which was shown in the column of $\mathrm{TN}^{\prime}$. For genotypes with $\mathrm{TN}>1$, we divided the number into integer and decimal directly; for genotypes with same number of TN, we added them together and then divided the result into integer and decimal; for genotypes with $\mathrm{TN}<1$, we also added them together and then divided the result. Then, we added 1 for genotypes with larger decimal part successively until the total number of isolates reach 30 , and they are shown in the column of $\mathrm{TN}^{\prime \prime}$. The final predicted frequency of genotype $(P r)$ was determined by dividing the number of each genotype (TN") by the total number of individuals $(n=30)$. Therefore, the predicted number of genotypes $(s)$ in this cultivar mixture was 11 . Thus, the theoretical $P G, E$, and Sh of Pst in mixture could be calculated based on the Pr, s, and $n$.

${ }^{\mathrm{b}}$ Frequencies of genotypes in $\mathrm{C} 1, \mathrm{C} 2$, and $\mathrm{C} 3$ were calculated as the number of each genotype divided by 34,31 , and 26 , respectively.

${ }^{c} \mathrm{TF}=$ the theoretical frequency of each genotype in cultivar mixture. For example, the predicted frequency for genotype g1 was $(0.47 \times 0.6+0 \times 0.2+0.04 \times 0.1) /$ $(0.6+0.2+0.1)=0.32$.

${ }^{\mathrm{d}} \mathrm{TN}^{\prime}=$ the theoretical number of genotypes which were divided into integer part and decimal part.

e $\mathrm{TN}^{\prime \prime}=$ the final theoretical number of genotypes in cultivar mixture, to which 1 was added for genotypes with a larger decimal part successively until the total number of isolates reached 30 .

f $\mathrm{Pr}=$ the final predicted frequency of each genotype in cultivar mixture after the determination of $\mathrm{TN}^{\prime \prime}$. 
(Fig. 1A and B). Specifically, the relative disease control efficacies of M1, M2, M3, and M9 that included the highly susceptible cultivar A were significantly lower than M4, M5, M6, M7, and M8 that did not include the highly susceptible cultivar A in the 2016-17 experiment based on Welch's $t$ test $(P=0.0418)$, while the relative disease control efficacies of M1, M2, M3, and M9 that included the highly susceptible cultivar A were significantly greater than M4, M5, M6, M7, and M8 that did not include the highly susceptible cultivar A in the 2017-18 experiment $(P=0.0159)$. This result indicated that the mixture effect was related to the resistance of the component cultivars and could be unstable in different epidemic years. The relative disease control efficacies of cultivar mixtures M2, M3, M4, M5, M6, M7, M8, and M9 were significantly higher than cultivar mixture M1 $(P<0.05)$ (Supplementary Fig. S1A). The relative disease control efficacies of M1, M2, M3, M6, and M7 were significantly higher than M4 in 2017-18 $(P<0.05)$ (Supplementary Fig. S1B). The average relative disease control efficacies of all cultivar mixtures were in $59.44 \%$ in $2016-17$ and $48.33 \%$ in $2017-18$.

No significant effects on relative yield increases were observed for all cultivar mixtures in the 2 years $(P<0.0056)$ (Fig. 1C and D). The relative yield increase efficacy of cultivar mixture M3 was significantly higher than M4, M5, M7, and M8 in the 2016-17 experiment but no significant differences were observed among the nine cultivar mixtures in the 2017-18 experiment $(P<0.05)$ (Supplementary Fig. S1C and D). The average relative yield increase efficacies for all cultivar mixtures were $8.37 \%$ in $2016-17$ and $5.34 \%$ in $2017-18$. Moreover, there was no significant correlation between the relative yield increase efficacy and relative disease control efficacy with cultivar mixtures $(P<0.05)$ (data not shown), suggesting that yield in cultivar mixtures is codetermined by many other factors in addition to stripe rust severity.

\section{Population genotypic diversity}

The 10 SSR loci yielded a total of 30 and 25 alleles ( 2 to 5 per locus) for our samples in the 2016-17 and 2017-18 experiments, respectively (Supplementary Table S2). Combining and comparing the multilocus genotypic diversity $(P G, E$, and $S h)$ among five pure stand populations showed that cultivar D had higher genotypic diversity than cultivars A, B, C, and E in 2016-17, and cultivars $\mathrm{B}, \mathrm{C}$, and $\mathrm{D}$ had higher genotypic diversity than $\mathrm{A}$ and $\mathrm{E}$ in 2017-18 (Fig. 2). Compared with the theoretical genotypic diversities, the four cultivar mixtures M1, M2, M4, and M5 in 2016-17 and five cultivar mixtures M3, M6, M7,
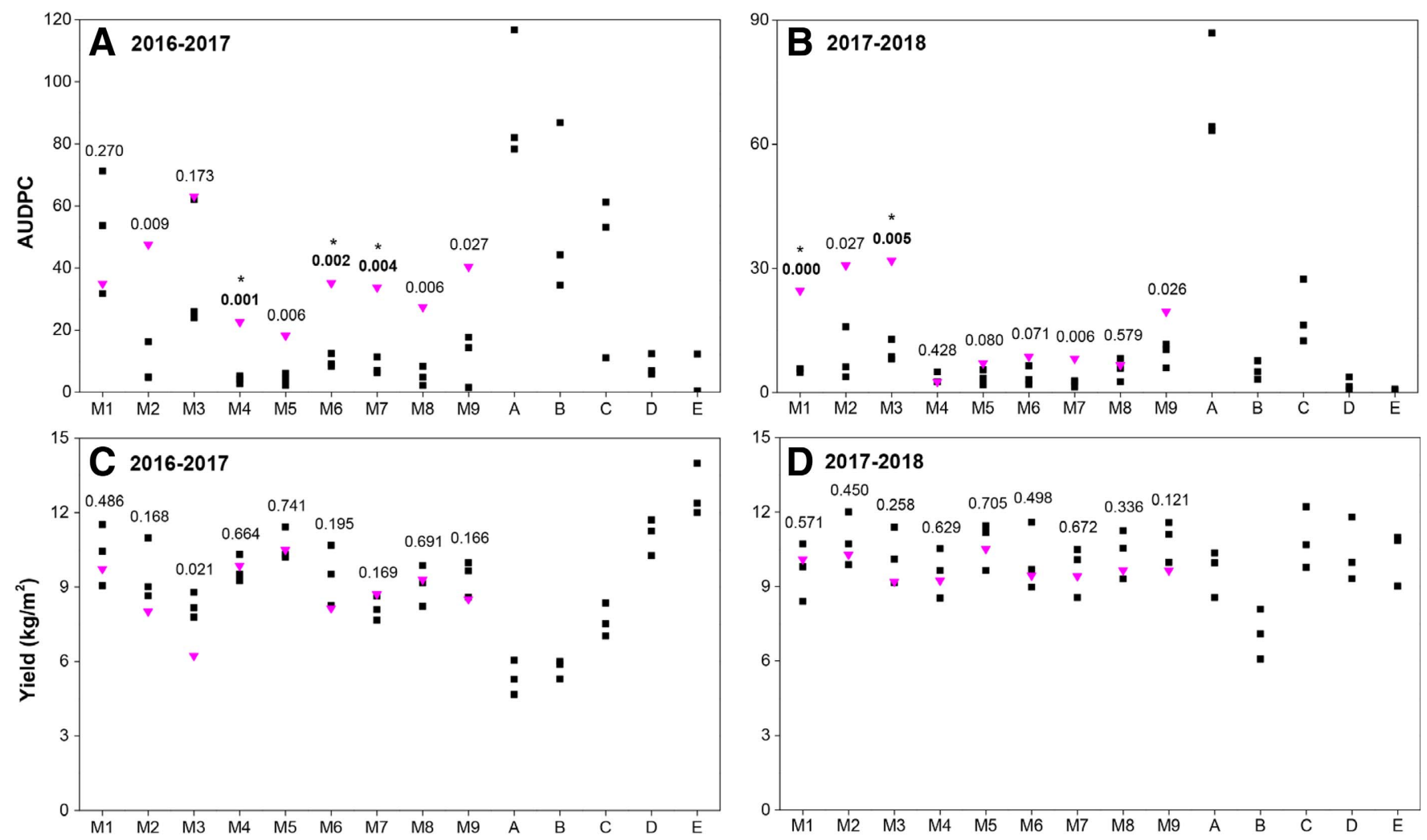

\section{Cultivar mixture treatment}

$M 1: A+D+E \quad M 2: A+C+D \quad M 3: A+B+C \quad M 4: B+D+E \quad M 5: C+D+E \quad M 6: B+C+D \quad M 7: B+C+E \quad M 8: B+C+D+E \quad M 9: A+B+C+D+E$ $\begin{array}{lllll}\text { A: Miannong } 4 \text { hao } & \text { B: Neimai } 9 \text { hao } & \text { C: Chuanmai } 51 & \text { D: Chuanyu } 21 & \text { E: Chuanmai } 55\end{array}$

- Observed value $\quad$ Theoretical value

FIGURE 1

Observed and theoretical area under the disease progress curve (AUDPC) of wheat stripe rust in nine cultivar mixtures and five pure stands in the A, 2016-17 and B, 2017-18 experiments, and the corresponding wheat yields in C, 2016-17 and D, 2017-18. Cultivar mixtures M1 to M9 were composed of different cultivars A, B, C, D, and E. All of the components in cultivar mixtures were mixed in equal proportions with a seed density of $16 \mathrm{~g} / \mathrm{m}^{2}$. Symbol colors: black represents observed AUDPC and yield in cultivar mixtures and monocultures and magenta represents the corresponding theoretical values in cultivar mixtures generated by averaging the observed monoculture AUDPC (or yield) for each component cultivar. The $P$ value of a one-sample $t$ test for each cultivar mixture is presented. $P$ values set in bold $\left({ }^{*}\right)$ indicated significant differences between observed values and corresponding theoretical values based on the Bonferroni correction $(P<0.0056)$. 
M8, and M9 in 2017-18 showed positive effects on the relative increase of Pst genotypic diversity. The two cultivar mixtures M7 and M9 in 2016-17 and one cultivar mixture M1 in 2017-18 showed negative effects based on all three measures of genotypic diversity. However, $P G, E$, and $S h$ were not consistent in M3, M6, and M8 in 2016-17 and M2, M4, and M5 in 2017-18 (Fig. 2; Supplementary Fig. S2A and B). Moreover, the effects of cultivar mixtures on the genotypic diversity of the Pst population were not consistent between the 2 years.

\section{Partition of genotypic variation among populations}

Based on the AMOVA (Table 4), significant genotypic differentiation among Pst populations from all pure stands and mixtures was observed: $P h i P T=0.213, P<0.001$ in $2016-17$ and $P h i P T=0.181, P<0.001$ in 2017-18. Moreover, the PhiPT of pure stand populations $(0.325, P<0.001)$ and $(0.241, P<$ 0.001 ) in 2016-17 and 2017-18, respectively, were higher than in the mixture populations $(0.149, P<0.001)$ and $(0.149$,
$P<0.001)$ in $2016-17$ and 2017-18, respectively, indicating a higher genotypic differentiation among populations of pure stands. Comparisons among pairwise populations showed significant genotypic differentiation $(P<0.05)$ for all combinations among monocultures and most combinations between mixtures and their component monocultures (Supplementary Table S3). However, low PhiPTs and no genotypic differentiation $(P>$ $0.05)$ were observed in combinations between the cultivar mixtures M4, M5, M6, M7, M8, and M9 and some corresponding component monocultures in 2017-18 (Supplementary Table S3, indicated by *), indicating a closer genetic relationship. Among them, five mixtures (M4, M5, M6, M7, and M8) were composed of resistant cultivars, and their effect on disease control was not as pronounced as mixtures that included the susceptible cultivar A. Mixtures M1, M2, M3, and M9 that included cultivar A in 2016-17 had the smallest PhiPT between M1 and cultivar A (0.103) among combinations of these mixtures and cultivar A (Supplementary Table S3). The relative disease control efficacy of the cultivar mixture M1 was the worst among these mixtures in 2016-17.

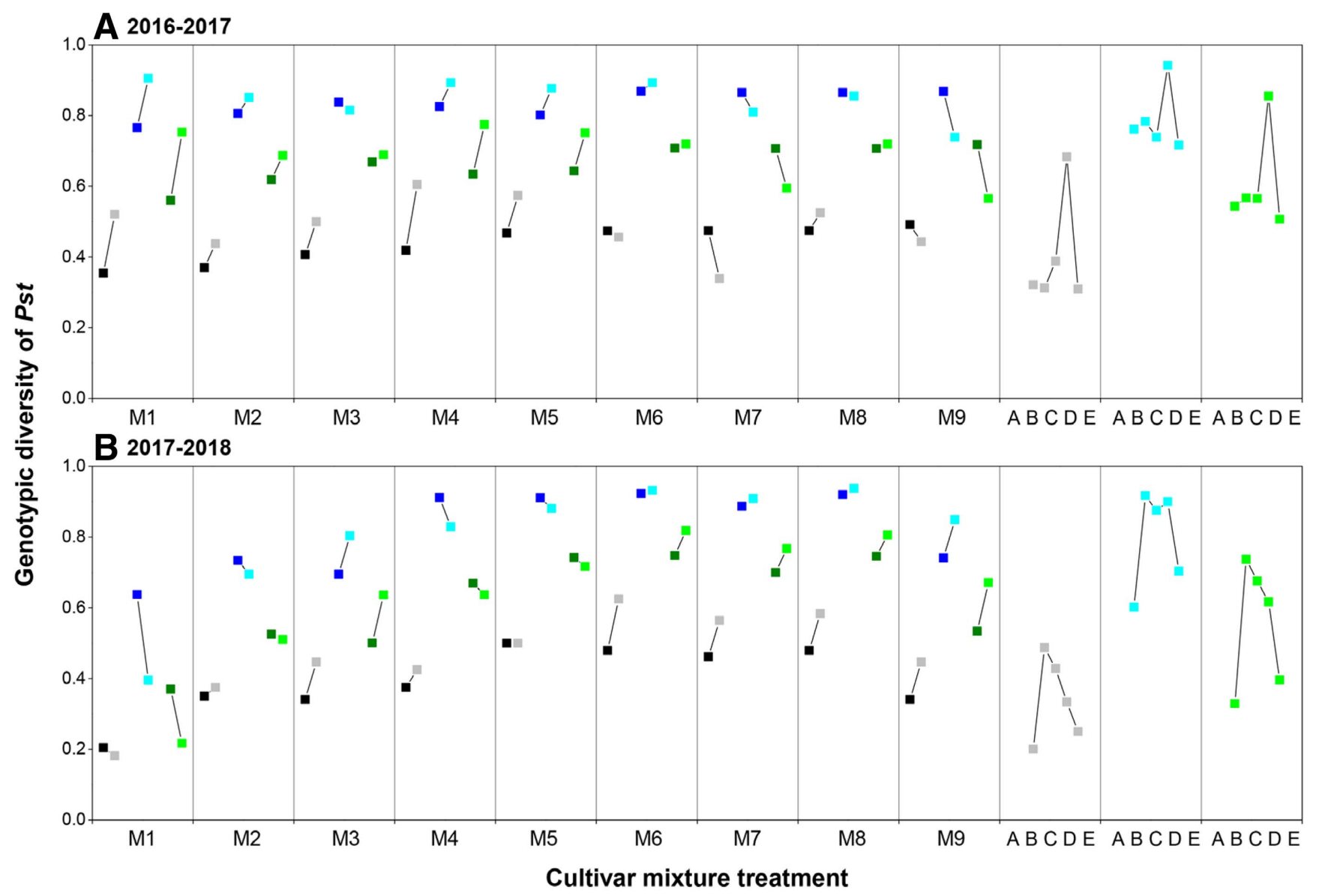

$M 1: A+D+E \quad M 2: A+C+D \quad M 3: A+B+C$ $A$ : Miannong 4 hao $\quad$ B: Neimai 9 hao

- Theoretical genotypic diversity $P G$

- Observed genotypic diversity $P G$
M4: $B+D+E \quad M 5: C+D+E \quad M 6: B+C+D \quad M 7: B+C+E$ $\begin{array}{ll}\text { C: Chuanmai } 51 & \text { D: Chuanyu } 21\end{array}$
- Theoretical genotypic diversity $E$

- Observed genotypic diversity $E$
- Theoretical genotypic diversity Sh

- Observed genotypic diversity Sh

\section{FIGURE 2}

Observed and theoretical genotypic diversity of Puccinia striiformis f. sp. tritici in nine cultivar mixtures and five pure stands in the A, 2016-17 and B, 2017-18 experiments. Cultivar mixtures M1 to M9 were composed of different cultivars A, B, C, D, and E. All of the components in cultivar mixtures were mixed in equal proportions with a seed density of $16 \mathrm{~g} / \mathrm{m}^{2}$. Symbol colors: black and gray represent theoretical and observed genotypic diversity proportion of multilocus genotypes $(P G)$, respectively; blue and cyan represent theoretical and observed genotypic diversity genotype evenness $(E)$, respectively; and olive and green represent theoretical and observed genotypic diversity Shannon's diversity index $(S h)$, respectively. 
The results of PCoA of five pure stands showed that, in 2016-17, cultivars A and B were almost contained in one and two quartiles, respectively, while cultivars C, D, and E were found in three, four, and four quartiles, respectively. In 2017-18, cultivar A was found in one quartile, while cultivars $\mathrm{B}, \mathrm{C}, \mathrm{D}$, and $\mathrm{E}$ were found in all four quartiles. Therefore, the PCoA distributions of Pst from highly and moderately susceptible cultivars were more centralized than those from resistant cultivars in the 2 years (Fig. 3A and B), indicating a closer genetic distance among Pst individuals from susceptible cultivars, consistent with the genotypic diversity findings. The PCoA distributions of the populations of all cultivar mixtures were equally or more dispersed compared with their component monocultures based on visual assessment of the 2 years of experiments (Supplementary Figs. S3 and S4), suggesting that cultivar mixtures increase the genetic distance among Pst individuals compared with monocultures. Moreover, compared with the mixtures composed of resistant cultivars (Supplementary Figs. S3C and S4B and C), the PCoA distributions of mixtures including cultivars A and B (highly susceptible and moderately susceptible, respectively) in 2016-17 (Supplementary Fig. S3A and B) and mixtures including cultivar A (highly susceptible) in 2017-18 (Supplementary Fig. S4A) were different from the corresponding monocultures of their most susceptible cultivar. This result implied a higher level of genetic variation between mixtures that included susceptible cultivars and monocultures of susceptible cultivars, consistent with the AMOVA results and pairwise comparisons.

\section{Genetic structuring of individuals}

The genetic similarity coefficients were 0.865 and 0.855 in 2016-17 and 2017-18, respectively. UPGMA cluster analyses revealed 835 and 568 individuals clustered into 11 groups termed P1 to P11 (2016-17) and P1' to P11' (2017-18), respectively (Fig. 4; Supplementary Fig. S5). The STRUCTURE results revealed that $\Delta K$ was maximized when $K=3$ and $K=$ 2 based on the rate of change in the log probability of data across successive $K$ values in 2016-17 and 2017-18, respectively (Evanno et al. 2005). To evaluate the population variance more precisely, the genetic grouping with $K=3$ in 2017-18 was also determined. Genotypes with $\mathrm{Q}>0.8$ were assigned to groups G1, G2, and G3 (2016-17) or G1', G2', and G3'
(2017-18), and genotypes with $0.2<\mathrm{Q}<0.8$ were considered to be admixed genotypes and assigned to the admixed group A (2016-17) or A' (2017-18) (Fig. 5). In the 2017-18 experiment, $\mathrm{G} 1^{\prime}$ was clustered in $K=2$, and $\mathrm{G} 2{ }^{\prime}$ and $\mathrm{G} 3{ }^{\prime}$ were clustered in only $K=3$ (data not shown).

Compared with monocultures of susceptible component cultivars (P1 and G1 of cultivar A and P2 and G2 of cultivar B in 2016-17, and $\mathrm{P} 1^{\prime}$ and $\mathrm{G} 1^{\prime}$ of cultivar $\mathrm{A}$ in 2017-18), the frequencies of $P$ st dominant genetic groups in cultivar mixtures that included the susceptible cultivars (M1, M2, M3, M4, M6, M7, M8, and M9 in 2016-17 and M1, M2, M3, and M9 in 2017-18) were all decreased (Figs. 4 and 5). Moreover, the frequencies of Pst dominant genetic groups (P1, P1', G1, and G1') of the highly susceptible cultivar A in all cultivar mixtures that included cultivar A (M1, M2, M3, and M9) were decreased compared with their corresponding theoretical frequencies in the 2 years, implying that cultivar mixtures could decrease the relative frequencies of $P s t$ dominant genetic groups of the highly susceptible component cultivar A (Tables 5 and 6). In contrast, the frequencies and relative frequencies of $P s t$ dominant genetic groups were not always decreased in cultivar mixtures that included their resistant component cultivars (Figs. 4 and 5; Tables 5 and 6). In addition, the reduced frequency of the Pst dominant genetic group of cultivar A was the same as the level of genotypic variation between Pst populations from mixtures and cultivar A, except that M3 > M9 according to the Bayesian model-based cluster analysis of the 2017-18 experiment (Tables 5 and 6; Supplementary Table S3). In particular, the cultivar mixture M1, which had negative disease control efficacy in the 2016-17 experiment, had the lowest reduction in relative frequencies of the Pst dominant genetic groups P1 and G1, consistent with the relatively low PhiPT between M1 and cultivar A (Tables 5 and 6; Supplementary Table S3).

\section{DISCUSSION}

Cultivar mixing has been successfully applied in disease management (Mundt 2002; Wolfe 1985; Zhu et al. 2000). Consistent with former research, our study showed that the AUDPCs of corresponding diseases in most cultivar mixtures were lower than the average AUDPCs of monocultures of component cultivars that were included in the mixtures, implying that positive

\section{TABLE 4}

Analysis of molecular variance (AMOVA) showing the partitioning of genotypic variation among and within populations of Puccinia striiformis f. sp. tritici sampled from 5 pure stands, 9 cultivar mixtures, and all 14 monoculture and mixture treatments ${ }^{\mathrm{a}}$

\begin{tabular}{|c|c|c|c|c|c|c|}
\hline Year, partitioning of variation & df & $\begin{array}{l}\text { Sum of } \\
\text { squares }\end{array}$ & $\begin{array}{l}\text { Variance } \\
\text { components }\end{array}$ & $\begin{array}{l}\text { Percentage of total } \\
\text { variation }(\%)\end{array}$ & PhiPT & $P$ \\
\hline \multicolumn{7}{|l|}{$2016-17$} \\
\hline Among 5 pure stand populations & 4 & 350.395 & 1.405 & 33 & 0.325 & $<0.001$ \\
\hline Within 5 pure stand populations & 297 & 866.592 & 2.918 & 67 & & \\
\hline Among 9 cultivar mixture populations & 8 & 277.864 & 0.538 & 15 & 0.149 & $<0.001$ \\
\hline Within 9 cultivar mixture populations & 524 & $1,603.916$ & 3.061 & 85 & & \\
\hline Among all 14 populations & 13 & 667.848 & 0.813 & 21 & 0.213 & $<0.001$ \\
\hline Within all 14 populations & 821 & $2,470.508$ & 3.009 & 79 & & \\
\hline \multicolumn{7}{|l|}{$2017-18$} \\
\hline Among 5 pure stand populations & 4 & 143.941 & 0.960 & 24 & 0.241 & $<0.001$ \\
\hline Within 5 pure stand populations & 168 & 508.898 & 3.029 & 76 & & \\
\hline Among 9 cultivar mixture populations & 8 & 223.934 & 0.565 & 15 & 0.149 & $<0.001$ \\
\hline Within 9 cultivar mixture populations & 386 & $1,247.097$ & 3.231 & 85 & & \\
\hline Among all 14 populations & 13 & 409.469 & 0.700 & 18 & 0.181 & $<0.001$ \\
\hline Within all 14 populations & 554 & $1,755.994$ & 3.170 & 82 & & \\
\hline
\end{tabular}

${ }^{\text {a }}$ Analyses were performed on the codominant genotypic distance matric of simple sequence repeat data with 999 permutations. 
disease control may not always exist in cultivar mixtures. Compared with the theoretical values, the frequencies of Pst dominant genetic groups of the highly susceptible component cultivar A were decreased in all cultivar mixtures studied. Pst populations in the cultivar mixtures had lower genotypic variation than those in monocultures. Although the statistical analysis on the genotypic diversity of Pst was not performed owing to the heavy workload of simple-spore separation and rust collections being bulked over replications in this study, observational results demonstrated the effect of cultivar mixtures on the population genetic structure of Pst and the differences between the mixtures.

\section{Factors affecting the effect of cultivar mixtures on disease control}

The effect of cultivar mixtures on disease control is affected by many factors (Mundt 2002). The outside inoculum and mixture composition had substantial effects on disease control of cultivar mixtures in our experiments. In contrast to travelingwave epidemics, the size of the initial outbreak focus is expected to have a large impact on accelerating-wave epidemics (Mundt et al. 2009a, b, 2011), and cultivar mixtures for these diseases are particularly vulnerable to the influence of the outside inoculum (Mundt 2002, 2014; Wolfe 1985). Indeed, we found that the disease control effects of mixtures containing highly susceptible and resistant cultivars were greater under normal inoculum pressure than under high inoculum pressure. This observation aligned with results obtained by Newton et al. (2002) and studies

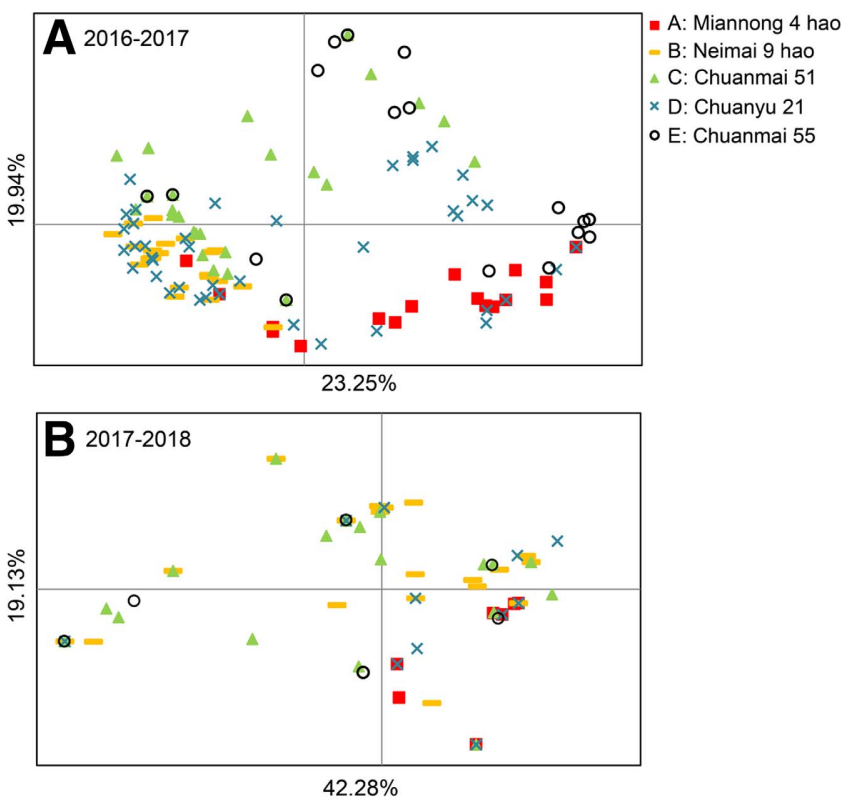

FIGURE 3

Results of the principal coordinate analysis on populations of Puccinia striiformis f. sp. tritici (Pst) sampled from five pure stands in the A, 2016-17 and B, 2017-18 experiments. Five pure stands were Miannong 4 hao, Neimai 9 hao, Chuanmai 51, Chuanyu 21, and Chuanmai 55 , coded as A, B, C, D, and E, respectively. Cultivars $A, B, C, D$, and $E$ were highly susceptible, moderately susceptible, moderately resistant, highly resistant, and highly resistant, respectively, to Pst in the 2016-17 experiment. Cultivar A was highly susceptible and cultivars $B, C, D$, and $E$ were resistant to Pst in the 2017-18 experiment. of the potato-Phytophthora infestans pathosystem (Garrett et al. 2001; Pilet et al. 2006). In our study, 2017 and 2018 were the pandemic and normal epidemic years of wheat stripe rust in China, when the inoculum pressures were high and normal, respectively. Under high inoculum pressure conditions, the availability of uninfected host tissue in monocultures of the highly susceptible cultivar A might limit disease progression, and the dilution effect in mixtures that included cultivar A might not work well (Keesing et al. 2006; Mundt 2002; Wolfe 1985). This observation suggested that the relative control efficacies of mixtures that included the highly susceptible cultivar A were worse than mixtures composed of moderately susceptible B and other resistant cultivars in 2016-17. By comparison, under normal inoculum pressure, mixtures composed of susceptible and resistant cultivars showed relatively higher disease control efficacies in 2017-18. Generally, the possibility of disease outbreaks of the level seen in 2017 in China is low; the previous wheat stripe rust pandemic was in 2002. In this situation, inoculum eradication by applying fungicide around the outbreak site at an early stage in the epidemic may be critical to reducing the disease outbreak (Mundt et al. 2011; Pilet et al. 2006).

The resistance levels of component cultivars might influence the mixture effects on disease reduction. In 2017-18, the relative control efficacies on AUDPC reduction of mixtures composed of resistant cultivars were lower than other mixtures, indicating that cultivar mixtures composed of resistant cultivars might benefit less from mixing, consistent with former studies (Guo 2012; Huang et al. 2012; McDonald et al. 1988). In particular, in 2017-18, cultivar mixture M4 showed a negative disease control effect despite being composed of three resistant cultivars B, D, and $\mathrm{E}$, which demonstrated low levels of disease when planted in monoculture. An antagonistic interaction between resistant cultivars might cause the disease increase in these mixtures (McDonald et al. 1988).

The negative disease control efficacy of M1 in 2016-17 is likely attributed to the competition of susceptible cultivar A, consistent with the observations of Alexander et al. (1986) and Finckh and Mundt (1992a). The mixture effect on disease control mainly results from epidemiological and selection effects (Finckh and Mundt 1992a; Garrett and Mundt 1999). Epidemiological effects lead to disease reduction in susceptible cultivars and disease increase or decrease in resistant cultivars, depending on the combination of race-specific and race-nonspecific resistance. However, race-nonspecific resistance generally happens between susceptible and moderately resistant cultivars (Garrett and Mundt 1999; Sapoukhina et al. 2013). Selection effects might benefit susceptible genotypes or resistant cultivars in different situations depending on the competition between them (Finckh and Mundt 1992a; Garrett and Mundt 1999). Therefore, a cultivar mixture will have a negative effect in two cases: (i) the increase in disease intensity in the resistant cultivars is greater than the decrease in disease intensity in the susceptible cultivar and (ii) plant competition benefits the susceptible component cultivar (Garrett and Mundt 1999). The increase in disease intensity of highly resistant cultivars under epidemiological effects rarely occurs; therefore, it is likely that the negative effect of the cultivar mixture M1 in 2016-17 was due to the competitive success of susceptible cultivar A. The relative increase in Pst genotypic diversity and decrease in Pst dominant genetic group frequency also support this explanation for the negative effect of the cultivar mixture M1. However, the negative effect of the cultivar mixture M1 was not observed in 2017-18. In addition to the impact of epidemiological effects, the change in competitiveness between susceptible and resistant cultivars in different 
disease conditions may be another contributing factor (Alexander and Holt 1998; Burdon et al. 1984; Finckh and Mundt 1992a).

\section{Effect of cultivar mixtures on Pst population and influencing factors}

The theoretical values for Pst genotypic diversity and frequency of Pst dominant genetic groups were calculated assuming that no effects influenced the Pst population after cultivar mixing. In this way, the comparison between the observed values and corresponding theoretical values reflects the effect of the cultivar mixture on the Pst population. In the calculation of the theoretical values, different components in the mixture can select the same or different pathogen genotypes; thus, simply averaging or summing the diversity scores of the component populations in monoculture is unreasonable. After comparing the Pst genotypes from different component cultivars, the calculation of theoretical genotypic diversity of Pst based on the prediction of the number and frequency of Pst genotypes in mixtures is more accurate. In addition, when mixing different cultivars, the theoretical genotypic diversity of Pst and theoretical frequencies of $P s t$ dominant genetic groups in mixtures are related to the proportion of isolates sampled from different mixture components. In this study, a single pustule was isolated from each leaf to ensure that the amount of Pst isolates sampled from different components was consistent with their disease incidences when mixed in equal proportions. Therefore, the theoretical genotypic diversity of Pst and theoretical frequency of Pst dominant genetic groups in mixtures should be calculated by considering disease incidences of different components when planted in monocultures. These calculation methods are scientifically more accurate to evaluate the effects of cultivar mixtures on the Pst genotypic diversity and frequency of Pst dominant genetic groups.

Compared with the theoretical values, the genotypic diversity of the Pst population might be increased or decreased. Instead, the frequencies of Pst dominant genetic groups of the highly susceptible component cultivar A were decreased in all mixtures studied. The frequency of pathogen genotypes in mixtures is difficult to estimate but is mainly affected by their fitness on host genotypes and the competition among different genotypes (Chin and Wolfe 1984; Lannou et al. 2005). In general, the simple Pst genotypes produced in the highly susceptible cultivar always propagated in the same cultivar, whereas Pst genotypes produced in resistant cultivars successively infected different cultivars (Lannou and Mundt 1996). Pst genotypic diversities from resistant cultivars may be higher than or as low as those from susceptible cultivars but the frequency of the Pst dominant genetic group from the highly susceptible cultivar is generally higher than those from resistant cultivars based on our and other studies (Wang 2017; Zhan et al. 2013). Therefore, in theory, the relative genotypic diversity and frequency of Pst dominant genetic groups in mixtures should depend on the ratio of randomly collected diseased leaves of susceptible to resistant cultivars in mixtures affected by mixture effects on disease control and the Pst population structure of resistant cultivars.

In most cases, epidemiological effects account for the main disease control (Finckh and Mundt 1992a; Garrett and Mundt

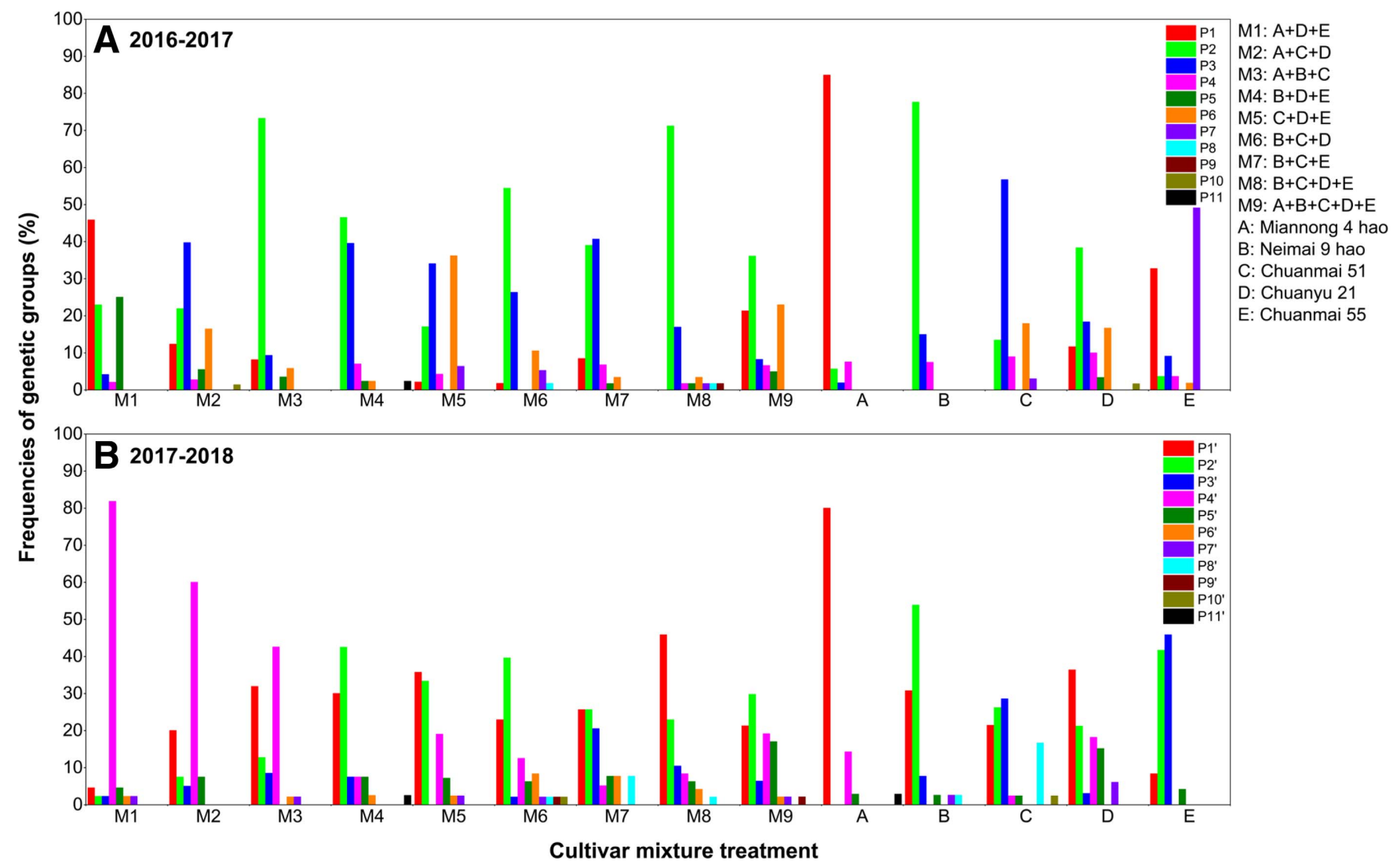

FIGURE 4

Frequencies of genetic groups of Puccinia striiformis $\mathrm{f}$. $\mathrm{sp}$. tritici in each cultivar mixture and pure stand based on the unweighted pair-group method with arithmetic mean cluster for 835 and 568 isolates collected from the A, 2016-17 and B, 2017-18 experiments, respectively. All of the components in cultivar mixtures were mixed in equal proportions. 
1999; Mundt 2002). The positive mixture effect will be observed when disease reduction is greater for the more susceptible genotype, and the negative mixture effect will occur when disease increase in resistant cultivars is greater than disease decrease in susceptible cultivars, although this is not common (Garrett and Mundt 1999). In both situations, the ratio of randomly collected diseased leaves from susceptible cultivars to resistant cultivars is decreased. Therefore, the relative frequencies of Pst dominant genetic groups in susceptible component cultivars in these mixtures could be decreased but the relative Pst genotypic diversities are related to the Pst genotypic diversities of resistant components in the mixtures and are not likely to be increased in mixtures composed of resistant cultivars with low Pst diversities. In our study, the mixtures that showed positive mixture effects (M4, M6, and M7 in 2016-17 and M1 and M3 in 2017-18) were probably due to disease reduction being greater for the more susceptible genotype, supported by the observations of the Pst population. Notably, no increase in relative genotypic diversities of Pst were observed in M6 and M8 in 2016-17. In addition to the previously described factors, a possible explanation is that race-nonspecific differences in resistance between cultivars $\mathrm{B}$ and $\mathrm{C}$ increased the incidence of cultivar $\mathrm{C}$ and, thus, decreased the diversity provided by cultivar $\mathrm{D}$ (more races were shared between cultivars $\mathrm{C}$ and D) (unpublished data).

Although epidemiological effects typically contribute the most to disease control, selection effects might play a major role in some mixtures (Finckh and Mundt 1992a). The positive mixture effect will be observed when competition benefits the resistant cultivar; the negative mixture effect will occur in situations where the susceptible cultivar competes successfully, although this is uncommon (Alexander et al. 1986; Finckh and Mundt 1992a, b). In the first case, the ratio of randomly collected diseased leaves of susceptible cultivars to resistant cultivars is decreased. Thus, the relative frequencies of the Pst dominant genetic groups of the susceptible cultivar in the mixture might be decreased but the Pst relative genotypic diversity is related to the Pst genotypic diversities of the resistant cultivars in the mixture. In the second case, the ratio of randomly collected diseased leaves of susceptible cultivars to resistant cultivars is increased. The relative frequencies of the Pst dominant genetic groups might be increased or decreased. An increase in the susceptible cultivar could increase the frequencies but infection of Pst from resistant cultivars to susceptible cultivars could decrease

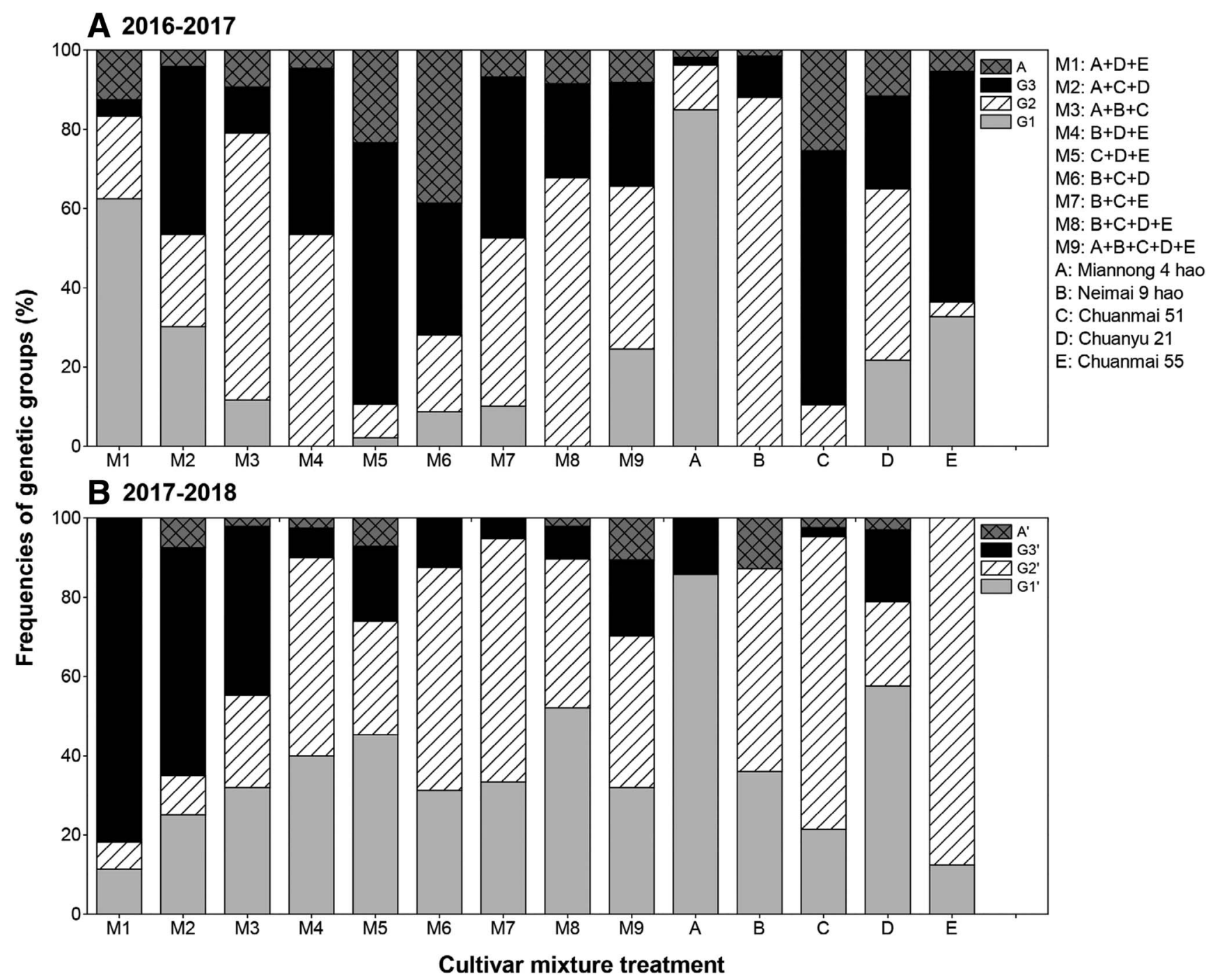

FIGURE 5

Bayesian assignments of Puccinia striiformis f. sp. tritici individuals for each cultivar mixture and pure stand in the A, 2016-17 and B, 2017-18 experiments. The proportion of individuals belonging to different groups of $G 1, \mathrm{G} 2$, G3, and A in 2016-17, and G1', G2' , G3', and A' in 2017-18 are indicated by different legends, with $Q>0.8$. All of the components in cultivar mixtures were mixed in equal proportions. 
the frequencies. Similarly, the relative genotypic diversity of Pst might be increased or decreased and is related to the Pst genotypic diversities of the resistant cultivars in addition to the above factors. In our study, cultivar mixture M1 in the 2016-17 experiment could probably be ascribed to the second case. A possible explanation is that the increase in Pst genotypic diversity caused by infection from cultivar D to cultivar A was greater than the decrease in Pst genotypic diversity caused by the successful competition of cultivar A. Also, the relative decrease in the frequency of Pst dominant genetic group was similar.

Collectively, our study demonstrated that the relative changes in Pst population genetic structure of cultivar mixtures were decided by the mixture effects on disease control and the Pst population structure of mixture components. In the condition with positive mixture effects on disease reduction, the relative frequencies of Pst dominant genetic groups were decreased whereas, in the condition with negative mixture effects, the relative frequencies of
Pst dominant genetic groups might be increased or decreased. Some previous simulation studies have demonstrated that the frequency of simple races is strongly limited when the mixture efficacy on disease control is high (Lannou and Mundt 1996; Østergaard 1983), consistent with our study. The genotypic diversities of Pst were increased in all cultivar mixtures compared with monocultures, consistent with previous observations in the riceM. grisea pathosystem (He et al. 2003; Yang et al. 2004; Zhu et al. 2004a). Pathogen diversity in cultivar mixtures benefits disease control and has been confirmed as "resistance enhancement" (Zhu et al. 2000) and "induced resistance" (Lannou et al. 1995). Although the increased Pst genotypic diversity in cultivar mixtures was not reflected in the pathogen diversity effect on disease control in our study, it did not mean that Pst diversity was not conducive to disease control. It suggested that the diversity effect was not the only mechanism of disease control, and this effect could be affected by other factors.

\section{TABLE 5}

Relative decreases in frequencies of dominant genetic groups of Puccinia striiformis f. sp. tritici (Pst) of each component monoculture in cultivar mixtures based on the unweighted pair-group method with arithmetic mean cluster in the 2016-17 and 2017-18 experiments ${ }^{\mathrm{a}}$

\begin{tabular}{|c|c|c|c|c|c|c|c|c|c|c|c|c|c|c|c|}
\hline \multirow[b]{4}{*}{ Treatment } & \multicolumn{15}{|c|}{ Relative decrease in frequency of Pst dominant genetic groups (\%) } \\
\hline & \multicolumn{6}{|c|}{$2016-17$} & \multicolumn{9}{|c|}{$2017-18$} \\
\hline & \multirow{2}{*}{$\frac{\mathrm{A}}{\mathrm{P} 1}$} & \multirow{2}{*}{$\frac{\mathrm{B}}{\mathrm{P} 2}$} & \multirow{2}{*}{$\frac{\mathrm{C}}{\mathrm{P} 3}$} & \multirow{2}{*}{$\frac{\mathrm{D}}{\mathrm{P} 2}$} & \multicolumn{2}{|c|}{$\mathrm{E}$} & \multirow{2}{*}{$\frac{\mathrm{A}}{\mathrm{P} 1^{\prime}}$} & \multicolumn{2}{|c|}{ B } & \multicolumn{3}{|c|}{$\mathrm{C}$} & \multirow{2}{*}{$\frac{\mathrm{D}}{\mathrm{P} 1^{\prime}}$} & \multicolumn{2}{|c|}{$\mathrm{E}$} \\
\hline & & & & & P1 & $\mathrm{P} 7$ & & $\mathrm{P} 1^{\prime}$ & $\mathrm{P} 2^{\prime}$ & $\mathrm{P} 1^{\prime}$ & $\mathrm{P} 2^{\prime}$ & $\mathrm{P}^{\prime}$ & & $\mathrm{P} 2^{\prime}$ & $\mathrm{P}^{\prime}$ \\
\hline M1 & 45 & - & - & -278 & 45 & 100 & 94 & - & - & - & - & - & 94 & 27 & -133 \\
\hline M2 & 81 & - & -192 & -187 & - & - & 70 & - & - & 70 & -23 & 22 & 70 & - & - \\
\hline M3 & 84 & -211 & 33 & - & - & - & 52 & 52 & -81 & 52 & -81 & -31 & - & - & - \\
\hline M4 & - & 37 & - & 37 & 100 & 100 & - & 9 & -50 & - & - & - & 9 & -50 & -2 \\
\hline M5 & - & - & 36 & -21 & -5 & -29 & - & - & - & -39 & -32 & 100 & -39 & -32 & 100 \\
\hline M6 & - & -7 & 18 & -7 & - & - & - & 14 & -49 & 14 & -49 & 89 & 14 & - & - \\
\hline M7 & - & 22 & -28 & - & $-1,173$ & 100 & - & -18 & 13 & -18 & 13 & 25 & - & 37 & 25 \\
\hline M8 & - & -43 & 46 & -43 & 100 & 22 & - & -77 & 15 & -77 & 15 & 49 & -77 & 15 & 49 \\
\hline M9 & 58 & -53 & 41 & -53 & 58 & 100 & 66 & 66 & -241 & 66 & -241 & 3 & 66 & -241 & 3 \\
\hline
\end{tabular}

${ }^{\text {a }}$ Frequencies were estimated as $\left[\left(c_{2}-c_{1}\right) / c_{2}\right] \times 100 \%$, where $c_{1}$ is the observed frequency of Pst dominant genetic group in cultivar mixture and $c_{2}$ is the corresponding theoretical frequency of the $P s t$ dominant genetic group, estimated as $\sum_{i}^{n} P_{i} x_{i} / \sum_{i}^{n} x_{i}$, where $n$ is the number of cultivars that included in mixture, $P_{i}$ is the frequency of $P s t$ dominant genetic group in $i$ th component monoculture, and $x_{i}$ is the disease incidence of $i$ th component monoculture on 21 March for the 2016-17 season and 29 March for the 2017-18 season. Bold text indicates the relative decreases in frequencies of Pst dominant genetic groups of the highly susceptible component cultivar A in all cultivar mixtures that included cultivar A.

TABLE 6

Relative decreases in frequencies of dominant genetic groups of Puccinia striiformis $\mathrm{f}$. sp. tritici (Pst) of each component monoculture in cultivar mixtures based on the model-based Bayesian cluster in the 2016-17 and 2017-18 experiments ${ }^{\mathrm{a}}$

\begin{tabular}{|c|c|c|c|c|c|c|c|c|c|c|c|c|}
\hline \multirow[b]{4}{*}{ Treatment } & \multicolumn{12}{|c|}{ Relative decrease in frequency of Pst dominant genetic groups (\%) } \\
\hline & \multicolumn{6}{|c|}{$2016-17$} & \multicolumn{6}{|c|}{$2017-18$} \\
\hline & \multirow{2}{*}{$\frac{\mathrm{A}}{\mathrm{G} 1}$} & \multirow{2}{*}{$\frac{\mathrm{B}}{\mathrm{G} 2}$} & \multirow{2}{*}{$\frac{\mathrm{C}}{\mathrm{G} 3}$} & \multirow{2}{*}{$\frac{\mathrm{D}}{\mathrm{G} 2}$} & \multicolumn{2}{|c|}{ E } & \multirow{2}{*}{$\frac{\mathrm{A}}{\mathrm{G} 1^{\prime}}$} & \multicolumn{2}{|c|}{ B } & \multirow{2}{*}{$\frac{\mathrm{C}}{\mathrm{G} 2^{\prime}}$} & \multirow{2}{*}{$\frac{\mathrm{D}}{\mathrm{G} 1^{\prime}}$} & \multirow{2}{*}{$\frac{\mathrm{E}}{\mathrm{G} 2^{\prime}}$} \\
\hline & & & & & G1 & G3 & & G1' & $\mathrm{G} 2^{\prime}$ & & & \\
\hline$\overline{\mathrm{M} 1}$ & 25 & - & - & -79 & 25 & -43 & 86 & - & - & - & 86 & -84 \\
\hline M2 & 55 & - & -178 & -103 & - & - & 65 & - & - & 40 & 65 & - \\
\hline M3 & 77 & -133 & 17 & - & - & - & 55 & 55 & -34 & -34 & - & - \\
\hline M4 & - & 36 & - & 36 & 100 & -236 & - & 20 & -57 & - & 20 & -57 \\
\hline M5 & - & - & -6 & 27 & 14 & -6 & - & - & - & 51 & -40 & 51 \\
\hline M6 & - & 65 & -2 & 65 & - & - & - & 6 & 0 & 0 & 6 & - \\
\hline M7 & - & 23 & -22 & - & $-1,428$ & -22 & - & -49 & 15 & 15 & - & 15 \\
\hline M8 & - & -24 & 28 & -24 & 100 & 28 & - & -60 & 35 & 35 & -60 & 35 \\
\hline M9 & 52 & -42 & -81 & -42 & 52 & -81 & 53 & 53 & -106 & -106 & 53 & -106 \\
\hline
\end{tabular}

${ }^{\mathrm{a}}$ Frequencies were estimated as $\left[\left(c_{2}-c_{1}\right) / c_{2}\right] \times 100 \%$, where $c_{1}$ is the observed frequency of Pst dominant genetic group in cultivar mixture and $c_{2}$ is the corresponding theoretical frequency of the $P s t$ dominant genetic group, estimated as $\sum_{i}^{n} P_{i} x_{i} / \sum_{i}^{n} x_{i}$, where $n$ is the number of cultivars that included in mixture, $P_{i}$ is the frequency of $P s t$ dominant genetic group in $i$ th component monoculture, and $x_{i}$ is the disease incidence of $i$ th component monoculture on 21 March for the 2016-17 season and 29 March for the 2017-18 season. Bold text indicates the relative decreases in frequencies of Pst dominant genetic groups of the highly susceptible component cultivar A in all cultivar mixtures that included cultivar A. 


\section{The role of cultivar mixtures on reducing the evolutionary potential of pathogens}

Aside from the short-term epidemiology purpose of cultivar mixtures on disease control, the long-term aim to reduce the evolutionary potential of pathogens is also significant for the use of cultivar mixtures (Zhan et al. 2015). In this study, genotypic variation among $P$ st populations from mixtures was lower than in monocultures, based on neutral genetic markers. However, this result did not directly address the idea that cultivar mixtures could reduce the directional selection on Pst populations by monocultures because there was no relationship between pathogen diversity of neutral markers and phenotypic traits (Chen et al. 1993; Zheng et al. 2000). Based on the theories of coevolutionary interactions between host plants and pathogens and trade-offs between pathogen infectivity and other life-history traits, selection on the Pst population in the cultivar mixtures is more disruptive than in monocultures of single resistant cultivars because host and environmental heterogeneity in cultivar mixtures can select for various pathogen phenotypes (McDonald and Linde 2002; Mundt 2002; J. Zhan et al. 2015). Therefore, it is beneficial for cultivar mixtures to delay the evolutionary potential of pathogens and reduce the opportunity for new infections (Thrall et al. 2011; J. Zhan et al. 2015). To avoid the emergence of superraces and their rapid increase in cultivar mixtures, the cultivar mixture composition needs to be changed after several seasons of use. In the future, the virulence of Pst should be detected directly or indirectly by genetic markers associated with Pst virulence patterns to help understand the effect of cultivar mixtures on pathogen virulence structure, which will provide further comprehensive suggestions for sustainable disease management and successful application of cultivar mixtures.

\section{Factors affecting the effect of cultivar mixtures on yield increase}

Previous work has shown that factors affecting mixture effects on yield increase mainly include disease stress, the number of component cultivars, and plant interactions, including competition, compensation, and complementation among component cultivars in mixtures (Mille et al. 2006; Smithson and Lenne 1996; Wolfe 1985). Although no significant relative increases in yield were observed in this study, cultivar mixtures showed improved yield stability relative to monocultures, especially in response to different years. Similar results for yield stability by cultivar mixtures were also observed in previous studies (Finckh et al. 2000; Mundt et al. 1995b). Owing to there being no significant correlation between the relative disease control efficacies and relative yield increase efficacies, we inferred that the relative yield variations in mixtures were mainly affected by the interactions among component cultivars. Finckh and Mundt (1992b) considered that yield compensation might obscure the effects of disease control on yield in mixtures. Interactions among component cultivars in mixtures were thought to be the major ecological process causing yield variation in mixtures (Callaway 1995; Finckh and Mundt 1992b; Mundt 2002; Smithson and Lenne 1996), and compensatory interactions accounted for the yield stability of mixtures rather than complementation (Creissen et al. 2013). In our study, it was interesting to find that cultivar mixture M3, composed of cultivars $\mathrm{A}, \mathrm{B}$, and $\mathrm{C}$, with high disease severities and low yield performances, had the best relative yield increase efficacy in 2016-17. There may be a strong compensation and complementation effect among these cultivars. Increasing and stabilizing yields are important goals of cultivar mixtures. Further studies should be conducted to focus on the interactions among different components of cultivar mixtures, which will play a considerable role in the selection of component cultivars.

This study described the effects of cultivar mixtures on wheat stripe rust disease and wheat yield using nine cultivar mixtures and estimated the population diversity and genetic structure of Pst in monocultures and mixtures through 10 pairs of SSR markers in a large sample size. These results are significant and useful for the application of cultivar mixtures and disease management.

\section{ACKNOWLEDGMENTS}

We thank X. Kang at Southwest University of Science and Technology and $\mathrm{X}$. Xu, X. Gong, W. Li, J. Fu, and Y. Zhang at the Plant Protection and Quarantine Station of Zitong County for their assistance in wheat planting, disease survey, sample collection, and yield measurement.

\section{LITERATURE CITED}

Alexander, H. M., and Holt, R. D. 1998. The interaction between plant competition and disease. Perspect. Plant Ecol. Evol. Syst. 1:206-220.

Alexander, H. M., Roelfs, A. P., and Cobbs, G. 1986. Effects of disease and plant competition on yield in monocultures and mixtures of two wheat cultivars. Plant Pathol. 35:457-465.

Andrivon, D., and de Vallavieille-Pope, C. 1995. Race diversity and complexity in selected populations of fungal biotrophic pathogens of cereals. Phytopathology 85:897-905.

Arnaud-Haond, S., and Belkhir, K. 2007. GenClone: A computer program to analyze genotypic data, test for clonality and describe spatial clonal organization. Mol. Ecol. Notes 7:15-17.

Bahri, B., Leconte, M., de Vallavieille-Pope, C., and Enjalbert, J. 2009. Isolation of ten microsatellite loci in an EST library of the phytopathogenic fungus Puccinia striiformis f. sp. tritici. Conserv. Genet. 10:1425.

Bahri, B., Shah, S. J. A., Hussian, S., Leconte, M., Enjalbert, J., and de Vallavieille-Pope, C. 2011. Genetic diversity of the wheat yellow rust population in Pakistan and its relationship with host resistance. Plant Pathol. 60:649-660.

Benesty, J., Chen, J., Huang, Y., and Cohen, I. 2009. Pearson correlation coefficient. Pages 1-4 in: Noise Reduction in Speech Processing. Springer Topics in Signal Processing, vol. 2. Springer, Berlin, Germany.

Burdon, J. J., Groves, R. H., Kaye, P. E., and Speer, S. S. 1984. Competition in mixtures of susceptible and resistant genotypes of Chondrilla juncea differentially infected with rust. Oecologia 64:199-203.

Callaway, R. M. 1995. Positive interactions among plants. Bot. Rev. 61:306-349.

Chen, C., Zheng, W., Buchenauer, H., Huang, L., Lu, N., and Kang, Z. 2009. Isolation of microsatellite loci from expressed sequence tag library of Puccinia striiformis f. sp. tritici. Mol. Ecol. Resour. 9:236-238.

Chen, Q., Zhu, Y., Li, Z., Kang, Y., and Kang, Z. 2009. Effect of wheat cultivar mixtures on wheat yield and stripe rust. Chin. J. Eco Agric. 17: 29-33.

Chen, X. 2005. Epidemiology and control of stripe rust [Puccinia striiformis f. sp. tritici] on wheat. Can. J. Plant Pathol. 27:314-337.

Chen, X., Line, R. F., and Leung, H. 1993. Relationship between virulence variation and DNA polymorphism in Puccinia striiformis. Phytopathology 83:1489-1497.

Chin, K. M., and Wolfe, M. S. 1984. Selection of Erysiphe graminis in pure and mixed stands of barley. Plant Pathol. 33:535-546.

Chu, B., Yang, L., Wang, C., Gu, Y., Yuan, K., Wang, R., Luo, Y., and Ma, Z. 2019. Improved evaluation of wheat cultivars (lines) on resistance to Puccinia striiformis f. sp. tritici using molecular disease index. Plant Dis. 103:1206-1212. 
Creissen, H. E., Jorgensen, T. H., and Brown, J. K. M. 2013. Stabilization of yield in plant genotype mixtures though compensation rather than complementation. Ann. Bot. (Lond.) 112:1439-1447.

Enjalbert, J., Duan, X., Giraud, T., Vautrin, D., de Vallavielle-Pope, C., and Solignac, M. 2002. Isolation of twelve microsatellite loci, using an enrichment protocol, in the phytopathogenic fungus Puccinia striiformis f. sp. tritici. Mol. Ecol. Notes 2:563-565.

Evanno, G., Regnaut, S., and Goudet, J. 2005. Detecting the number of clusters of individuals using the software STRUCTURE: A simulation study. Mol. Ecol. 14:2611-2620.

Finckh, M. R., Gacek, E. S., Goyeau, H., Lannou, C., Merz, U., Mundt, C. C., Munk, L., Nadziak, J., Newton, A. C., de Vallavieille-Pope, C., and Wolfe, M. S. 2000. Cereal variety and species mixtures in practice, with emphasis on disease resistance. Agronomie 20:813-837.

Finckh, M. R., and Mundt, C. C. 1992a. Stripe rust, yield, and plant competition in wheat cultivar mixtures. Phytopathology 82:905-913.

Finckh, M. R., and Mundt, C. C. 1992b. Plant competition and disease in genetically diverse wheat populations. Oecologia 91:82-92.

Finckh, M. R., and Wolfe, M. S. 2006. Diversification strategies. Pages 269307 in: The Epidemiology of Plant Diseases. B. M. Cooke, D. G. Jones, and B. Kaye, eds. Springer, Berlin, Germany.

Garrett, K. A., and Mundt, C. C. 1999. Epidemiology in mixed host populations. Phytopathology 89:984-990.

Garrett, K. A., Nelson, R. J., Mundt, C. C., Chacon, G., Jaramillo, R. E., and Forbes, G. A. 2001. The effects of host diversity and other management components on epidemics of potato late blight in the humid highland tropics. Phytopathology 91:993-1000.

Goodwin, S. B., Spielman, L. J., Matuszak, J. M., Bergeron, S. N., and Fry, W. E. 1992. Clonal diversity and genetic differentiation of Phytophthora infestans populations in northern and central Mexico. Phytopathology 82: 955-961.

Guo, S. 2012. Studies on the use of wheat cultivar mixtures for the control of stripe rust. Doctoral dissertation, Northwest A\&F University, Yangling, China.

He, X., Yang, J., Wang, Y., Zhou, H., Chen, J., Li, Z., Li, Y., and Zhu, Y. 2003. Analysis of genetic structure of Magnaporthe grisea in the fields of different rice varieties. Chin. J. Appl. Ecol. 14:733-736.

Huang, C., Sun, Z., Wang, H., Luo, Y., and Ma, Z. 2012. Effects of wheat cultivar mixtures on stripe rust: A meta-analysis on field trails. Crop Prot. 33:52-58.

Jakobsson, M., and Rosenberg, N. A. 2007. CLUMPP: A cluster matching and permutation program for dealing with label switching and multimodality in analysis of population structure. Bioinformatics 23: 1801-1806.

Jensen, R. J. 1989. NTSYS-PC. Q. Rev. Biol. 64:250-252.

Jia, S. 2007. The effects of wheat cultivar mixtures on Blumeria graminis $\mathrm{f}$. sp. tritici and its population structure. Master dissertation, Fujian A\&F University, Fuzhou, China.

Kang, Z., Wang, X., Zhao, J., Tang, C., and Huang, L. 2015. Advances in research of pathogenicity and virulence variation of the wheat stripe rust fungus Puccinia striiformis f. sp. tritici. Sci. Agric. Sin. 48:34393453.

Keesing, F., Holt, R. D., and Ostfeld, R. S. 2006. Effects of species diversity on disease risk. Ecol. Lett. 9:485-498.

Lannou, C., de Vallavieille-Pope, C., and Goyeau, H. 1995. Induced resistance in host mixtures and its effect on disease control in computer-simulated epidemics. Plant Pathol. 44:478-489.

Lannou, C., Hubert, P., and Gimeno, C. 2005. Competition and interactions among stripe rust pathotypes in wheat-cultivar mixtures. Plant Pathol. 54: 699-712.

Lannou, C., and Mundt, C. C. 1996. Evolution of a pathogen population in host mixtures: Simple race-complex race competition. Plant Pathol. 45: 440-453.

Li, Z., and Zeng, S. 2002. Wheat Rust in China. China Agriculture Press, Beijing, China.

Liu, B., Liu, T., Zhang, Z., Jia, Q., Wang, B., Gao, L., Peng, Y., Jin, S., and Chen, W. 2017. Discovery and pathogenicity of CYR34, a new race of Puccinia striiformis f. sp. tritici in China. Acta Phytopathol. Sin. 47: 681-687.
Liu, T., Peng, Y., Chen, W., and Zhang, Z. 2010. First detection of virulence in Puccinia striiformis f. sp. tritici in China to resistance genes $Y r 24$ $(=Y r 26)$ present in wheat cultivar Chuanmai 42. Plant Dis. 94:1163.

Luo, Y., Ma, Z., and Ma, Z. 2007. Introduction to Molecular Epidemiology of Plant Diseases. China Agriculture Press, Beijing, China.

McDonald, B. A., Allard, R. W., and Webster, R. K. 1988. Responses of two-, three-, and four-component barley mixtures to a variable pathogen population. Crop Sci. 28:447-452.

McDonald, B. A., and Linde, C. 2002. Pathogen population genetics, evolutionary potential, and durable resistance. Annu. Rev. Phytopathol. 40:349-379.

McDonald, J. H. 2014a. Student's $t$-test for one sample. Pages 121-125 in: Handbook of Biological Statistics, 3rd ed. Sparky House Publishing, Baltimore, MD.

McDonald, J. H. 2014b. Student's $t$-test for two samples. Pages 126-130 in: Handbook of Biological Statistics, 3rd ed. Sparky House Publishing, Baltimore, MD.

McDonald, J. H. 2014c. One-way anova. Pages 145-156 in: Handbook of Biological Statistics, 3rd Ed. Sparky House Publishing, Baltimore, MD.

Mille, B., Fraj, M. B., Monod, H., and de Vallavieille-Pope, C. 2006. Assessing four-way mixtures of winter wheat cultivars from the performances of their two-way and individual components. Eur. J. Plant Pathol. 114:163-173.

Müller, K., McDermott, J. M., Wolfe, M. S., and Limpert, E. 1996. Analysis of diversity in populations of plant pathogens: The barley powdery mildew pathogen across Europe. Eur. J. Plant Pathol. 102:385-395.

Mundt, C. C. 1994. Use of host genetic diversity to control cereal diseases: Implications for rice blast. Pages 293-308 in: Rice Blast Disease. R. S. Zeigler, S. A. Leong, and P. S. Teng, eds. CAB International, Wallingford, U.K.

Mundt, C. C. 2002. Use of multiline cultivars and cultivar mixtures for disease management. Annu. Rev. Phytopathol. 40:381-410.

Mundt, C. C. 2014. Durable resistance: A key to sustainable management of pathogens and pests. Infect. Genet. Evol. 27:446-455.

Mundt, C. C., Brophy, L. S., and Schmitt, M. S. 1995a. Choosing crop cultivars and cultivar mixtures under low versus high disease pressure: A case study with wheat. Crop Prot. 14:509-515.

Mundt, C. C., Brophy, L. S., and Schmitt, M. S. 1995b. Disease severity and yield of pure-line wheat cultivars and mixtures in the presence of eyespot, yellow rust, and their combination. Plant Pathol. 44:173-182.

Mundt, C. C., Sackett, K. E., and Wallace, L. D. 2011. Landscape heterogeneity and disease spread: Experimental approaches with a plant pathogen. Ecol. Appl. 21:321-328.

Mundt, C. C., Sackett, K. E., Wallace, L. D., Cowger, C., and Dudley, J. P. 2009a. Aerial dispersal and multiple-scale spread of epidemic disease. EcoHealth 6:546-552.

Mundt, C. C., Sackett, K. E., Wallace, L. D., Cowger, C., and Dudley, J. P. 2009b. Long distance dispersal and accelerating waves of disease: Empirical relationships. Am. Nat. 173:456-466.

Newton, A. C., Ellis, R. P., Hackett, C. A., and Guy, D. C. 1997. The effect of component number on Rhynchosporium secalis infection and yield in mixtures of winter barley cultivars. Plant Pathol. 46:930-938.

Newton, A. C., Guy, D. C., Nadziak, J., and Gacek, E. S. 2002. The effect of inoculum pressure, germplasm selection and environment on spring barley cultivar mixtures efficacy. Euphytica 125:325-335.

Østergaard, H. 1983. Predicting development of epidemics on cultivar mixtures. Phytopathology 73:166-172.

Peakall, R., and Smouse, P. E. 2006. GENALEX 6: Genetic analysis in Excel. Population genetic software for teaching and research. Mol. Ecol. Notes 6:288-295.

Peakall, R., and Smouse, P. E. 2012. GenAlEx 6.5: Genetic analysis in Excel. Population genetic software for teaching and research-An update. Bioinformatics 28:2537-2539.

Pilet, F., Chacon, G., Forbes, G. A., and Andrivon, D. 2006. Protection of susceptible potato cultivars against late blight in mixtures increases with decreasing disease pressure. Phytopathology 96:777-783.

Pritchard, J. K., Stephens, M., and Donnelly, P. 2000. Inference of population structure using multilocus genotype data. Genetics 155:945959.

Rosenberg, N. A. 2004. Distruct: A program for the graphical display of population structure. Mol. Ecol. Notes 4:137-138. 
Sapoukhina, N., Paillard, S., Dedryver, F., and de Vallavieille-Pope, C. 2013. Quantitative plant resistance in cultivar mixtures: Wheat yellow rust as a modelling case study. New Phytol. 200:888-897.

Savary, S., Willocquet, L., Pethybridge, S. J., Esker, P., McRoberts, N., and Nelson, A. 2019. The global burden of pathogens and pests on major food crops. Nat. Ecol. Evol. 3:430-439.

Schachtel, G. A., Dinoor, A., Herrmann, A., and Kosman, E. 2012. Comprehensive evaluation of virulence and resistance data: A new analysis tool. Plant Dis. 96:1060-1063.

Shang, H., Ren, W., and Wang, S. 1990. The discussion of a grading standard of wheat stripe rust severity. Plant Prot. 16:31.

Sheldon, A. L. 1969. Equitability indices: Dependence on the species count. Ecology 50:466-467.

Smithson, J. B., and Lenne, J. M. 1996. Varietal mixtures: A viable strategy for sustainable productivity in subsistence agriculture. Ann. Appl. Biol. 128:127-158

Stubbs, R. W. 1985. Stripe rust. Pages 61-101 in: The Cereal Rusts II. A. P. Roelfs and W. R. Bushnell, eds. Academic Press, Orlando, FL.

Thrall, P. H., Oakeshott, J. G., Fitt, G., Southerton, S., Burdon, J. J., Sheppard, A., Russell, R. J., Zalucki, M., Heino, M., and Denison, R. F. 2011. Evolution in agriculture: The application of evolutionary approaches to the management of biotic interactions in agroecosystems. Evol. Appl. 4:200-215.

Tukey, J. 1953. Multiple comparisons. J. Am. Stat. Assoc. 48:624-625.

Wan, A. 2003. Differentials and nomenclature of races of Puccinia striiformis West. f. sp. tritici Eriksson. Acta Phytopathol. Sin. 33:481-486.

Wan, A., Chen, X., and He, Z. 2007. Wheat stripe rust in China. Aust. J. Agric. Res. 58:605-619.

Wan, Q., Liang, J., Luo, Y., and Ma, Z. 2015. Population genetic structure of Puccinia striiformis in northwestern China. Plant Dis. 99:1764-1774.

Wang, S. 2017. Population Genetic Structure and Parasitic Fitness of Puccinia striiformis f. sp. tritici in Sichuan Province of China. China Agricultural University, Beijing, China.

Wolfe, M. S. 1985. The current status and prospects of multiline cultivars and variety mixtures for disease resistance. Annu. Rev. Phytopathol. 23:251-273.
Xing, J., and Tian, L. 2011. Summary of calculate wheat yield methods. Anhui Agric. Sci. 17:94-95.

Yang, J., He, X., Wang, Y., Li, Z., and Zhu, Y. 2004. Study on constitution of physiological race of Magnaporthe grisea in rice mixture field. Acta Phytopathol. Sin. 34:468-471.

Zadoks, J. C., Chang, T. T., and Konzak, C. F. 1974. A decimal code for the growth stages of cereals. Weed Res. 14:415-421.

Zhan, G., Wang, F., Luo, H., Jiang, S., Zheng, W., Huang, L., and Kang, Z. 2015. Screening for simple sequence repeat markers in Puccinia striiformis tritici based on genomic sequence. J. Zhejiang Univ. Sci. B 16:727-732.

Zhan, G., Zhuang, H., Wang, F., Wei, G., Huang, L., and Kang, Z. 2013. Population genetic diversity of Puccinia striiformis f. sp. tritici on different wheat varieties in Tianshui, Gansu Province. World J. Microbiol. Biotechnol. 29:173-181.

Zhan, J., Thrall, P. H., Papaix, J., Xie, L., and Burdon, J. J. 2015. Playing on a pathogen's weakness: Using evolution to guide sustainable plant disease control strategies. Annu. Rev. Phytopathol. 53:19-43.

Zhang, Z., He, X., Wang, Y., Yang, J., Zhou, J., Li, Z., and Zhu, Y. 2004. Genetic structure of Magnaporthe grisea in the fields of different rice varieties in Yunnan japonica rice planted area. Chinese. J. Rice Sci. 18: 346-350.

Zheng, W., Liu, F., Kang, Z., Chen, S., Li, Z., and Wu, L. 2000. AFLP fingerprinting analysis of main epidemic strains of wheat stripe rust in China. Prog. Nat. Sci. 10:532-537.

Zhu, Y., Chen, H., Fan, J., Wang, Y., Li, Y., Chen, J., Fan, J., Yang, S., Hu, L., Leung, H., Mewk, T. W., Tengk, P. S., Wang, Z., and Mundt, C. C. 2000. Genetic diversity and disease control in rice. Nature 406:718-722.

Zhu, Y., Leung, H., Chen, H., Wang, Y., Tang, K., Zhao, X., Zhou, J., Tu, J., Li, Y., He, X., Zhou, J., Sun, Y., and Mew, T. W. 2004a. Using resistance genes diversity for sustainable rice disease control. Sci. Agric. Sin. 37:832-839.

Zhu, Y., Sun, Y., Wang, Y., Li, Y., He, Y., He, X., Mundt, C. C., Mew, T. W., and Leung, H. 2004b. Genetic analysis of rice varietal diversity for rice blast control. Acta Genet. Sin. 31:707-716. 